University of New Mexico

UNM Digital Repository

Electrical and Computer Engineering ETDs

Engineering ETDs

Spring 5-12-2018

\title{
On Improved Accuracy Chirp Parameter Estimation using the DFRFT with Application to SAR-based Vibrometry
}

Satish Mandal

Follow this and additional works at: https://digitalrepository.unm.edu/ece_etds

Part of the Electrical and Computer Engineering Commons

\section{Recommended Citation}

Mandal, Satish. "On Improved Accuracy Chirp Parameter Estimation using the DFRFT with Application to SAR-based Vibrometry." (2018). https://digitalrepository.unm.edu/ece_etds/407

This Thesis is brought to you for free and open access by the Engineering ETDs at UNM Digital Repository. It has been accepted for inclusion in Electrical and Computer Engineering ETDs by an authorized administrator of UNM Digital Repository. For more information, please contact disc@unm.edu. 


\section{Satish Mandal}

Candidate

\section{Electrical and Computer Engineering}

Department

This thesis is approved, and it is acceptable in quality and form for publication:

Approved by the Thesis Committee:

Dr. Balu Santhanam

, Chairperson

Dr. Majeed M. Hayat

Dr. Manel Martinez-Ramon 


\title{
ON IMPROVED ACCURACY CHIRP PARAMETER ESTIMATION USING THE DFRFT WITH APPLICATION TO SAR-BASED VIBROMETRY
}

\author{
by \\ Satish Mandal \\ B.S., ELECTRONICS AND COMMUNICATION, \\ TRIBHUVAN UNIVERSITY, NEPAL, 2014
}

\section{THESIS}

Submitted in Partial Fulfillment of the

Requirements for the Degree of

Master of Science

Electrical Engineering

The University of New Mexico

Albuquerque, New Mexico

May, 2018 


\section{Dedication}

I dedicate this work to my parents for their support and encouragement.

"I respect faith, but doubt is what gets you an education"

- Wilson Mizner 


\section{Acknowledgments}

First of all, I would like to express my deep and sincere gratitude to my advisor and thesis chair, Dr. Balu Santhanam, for providing me this great research opportunity, for giving me the professional and insightful comments and suggestion on my research work, and for encouraging and motivating me to move forward on the research. As an advisor, he taught me how to start a research project, and how to face challenges during the research work.

Besides my advisor, I would also like to express gratitude to my committee members: Dr. Majeed M. Hayat, and Dr. Manel Martinez-Ramon. Thank you for reviewing my thesis, participating on my defense, and for asking me challenging questions and giving me professional comments on my thesis.

Most importantly, I would like to express my deepest gratitude to my parents for their never-ending support and encouragement, to my brothers and sister, who has been there to boost me up whenever I am in difficulties.

I would also like to thank Dr. Mark Gilmore, Ex-director of the ECE Graduate Program, for appointing me as a Project Assistant at ECE Department for several semesters. This position helped me gain some experience in working with students.

I would also like to thank Francisco Perez-Venegas for helping me out numerous times during the course of research work.

Finally, I would also like to thank all of my friends at UNM. Because of you, the last two and half years at UNM have provided me with some of my most memorable moments in life.

This work has been supported by the U.S. Department of Energy under the Award DE-NA0002494. 


\title{
On Improved Accuracy Chirp Parameter Estimation using the DFRFT with Application to SAR-based Vibrometry
}

\author{
by
}

\author{
Satish Mandal \\ B.S., Electronics and Communication, Tribhuvan University, Nepal, 2014 \\ M.S., Electrical Engineering, University of New Mexico, 2018
}

\begin{abstract}
The Discrete Fractional Fourier Transform (DFRFT) has in recent years, become a useful tool for multicomponent chirp signal analysis. Chirp signals are transformed into spectral peaks in the chirp rate versus center frequency representation, whose coordinates are related to the underlying chirp parameters via a computed empirical peak to parameter mapping incorporated into the Santhanam-Peacock algorithm.
\end{abstract}

In this thesis, we attempt to quantify the accuracy of the DFRFT approach by first studying the discretization error sources that arise from the transitioning of the continuous FRFT to DFRFT. Then, we refine prior work by Ishwor Bhatta to develop analytical expressions for the chirp rate and center frequency parameters instead of the empirical mapping approach. We further study the extensions of this refined DFRFT approach using zero padding, spectral peak interpolation, and chirp-z-transform based zooming. The performance of the refined estimators is compared versus the Cramer-Rao lower bound and shown to asymptotically approach the bound. 
This refined DFRFT approach is then applied to Synthetic Aperture Radar Vibrometry data from several vibrating targets and the estimated acceleration information and vibration frequencies are shown to be very close to the corresponding ground-truth accelerometer measurements. 


\section{Contents}

List of Figure $\quad$ x

List of Tables $\quad$ xii

Abbreviations $\quad$ xiii

1 Introduction 1

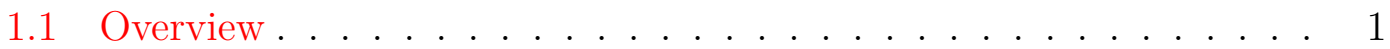

1.2 Fractional Fourier Transform . . . . . . . . . . . . . . . . . . 3

1.3 Discrete Fractional Fourier Transform . . . . . . . . . . . . . . . . . 4

1.4 Discrete Rotational Fourier Transform . . . . . . . . . . . . 5

1.5 Cramer-Rao Lower Bound . . . . . . . . . . . . . . . . . . . 6

1.6 Organization of the thesis . . . . . . . . . . . . 7

2 Effects of Windowing $\quad 9$

2.1 Empirical Estimation \& Subspace Decomposition . . . . . . . . . 9

2.2 Distortion Sources . . . . . . . . . . . . . . . . . . 11

2.3 Sources of Discretization Errors . . . . . . . . . . . . . . . 11 
2.4 QMFD Approach : Diagonal Q and Quasi-Toeplitz Matrices . . . . 13

2.5 QMFD Approach: Non-Diagonal Case . . . . . . . . . . . . . 15

2.6 Conclusion . . . . . . . . . . . . . . . . . . 20

3 Analytical Expressions for DFRFT Chirp Parameter Estimates 22

3.1 Multi-angle Centered FRFT . . . . . . . . . . . . . . . . 23

3.2 Linear Chirp Signal . . . . . . . . . . . . . . . . . . . . . . . 24

3.3 Relation between Peak and Parameter . . . . . . . . . . . . . . 25

3.4 MA-CDFRFT: Zero padding, Spectral Peak Interpolation and CZT

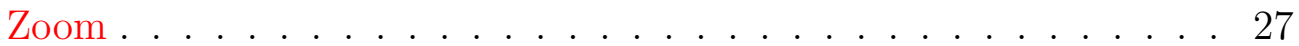

3.4.1 Parameter Estimation via the CDFT and Zero padding . . . 27

3.4.2 Parameter Estimation via the CDFT and Spectral Peak In-

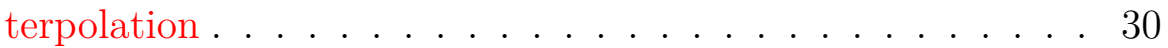

3.4.3 Parameter Estimation via the CDFT and CZT Zoom . . . . 33

3.4.4 Combination of the Refinements . . . . . . . . . . . . 34

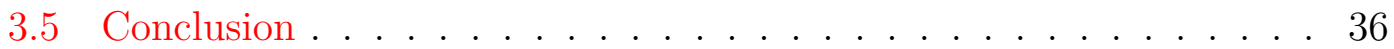

4 Application to Real SAR Data 38

4.1 Introduction . . . . . . . . . . . . . . . 38

4.2 SAR Signal Model . . . . . . . . . . . . . . . . . . . 40

4.3 Vibration Estimation . . . . . . . . . . . . . . . . . 40

4.4 Description of the Targets . . . . . . . . . . . . . . . . . 41

4.4.1 Top-hat chimney reflector . . . . . . . . . . . . . 41 
4.4.2 Rocking quad-corner reflector . . . . . . . . . . . . . . 42

4.4.3 Sliding quad-corner reflector . . . . . . . . . . . . . 43

4.5 Estimation using the Analytical Expressions . . . . . . . . . . . . 44

4.6 Conclusion . . . . . . . . . . . . . . . . . . . . . . . . 48

5 Conclusions and Future Work $\quad 50$

5.1 Future Work . . . . . . . . . . . . . . . . . 51

A Parameter to Peak Mapping 53

B Parameter Estimation using Analytical expressions and Subspace Decomposition

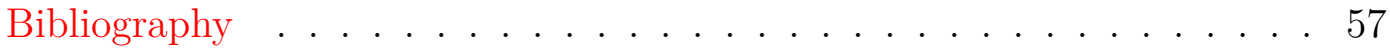




\section{List of Figures}

2.1 Effect of windowing on the eigenvalue spectrum. . . . . . . . . 16

2.2 Peak to parameter mappings for $N=256 \ldots \ldots \ldots$. . . . . 17

2.3 Effect of windowing eigenvalue sequence . . . . . . . . . . . . . . . 19

2.4 Invertibility percentage improvement . . . . . . . . . . . . 20

2.5 Impact of windowing on the MSE of parameter estimates . . . . . . 21

3.1 Graphical representation of $\mathbf{X}_{k} \ldots \ldots \ldots . \ldots 24$

3.2 Magnitude of the MA-CDFRFT for complex chirp signal . . . . . 25

3.3 Magnitude of the MA-CDFRFT for real chirp signal. . . . . . . . 26

3.4 Performance of the chirp parameter estimators based on the analytical expression and zero padding . . . . . . . . . . . . . 29

3.5 Effects of spectral peak interpolation on chirp parameter estimates . 32

3.6 Effect of CZT-based zoom on the chirp parameter estimates . . . . 34

3.7 Combined effects of zero padding and spectral peak interpolation . 34

3.8 Combined effects of zero padding and CZT zoom . . . . . . . . 35

3.9 Combined effects of zero padding and CZT zoom and speactral peak interpolation . . . . . . . . . . . . . . . . 36 
3.10 Comparision between the vanilla analytical expression and the final analytical expression with the refinements . . . . . . . . 37

4.1 Graphical design of the top-hat chimney reflector . . . . . . . . . . 42

4.2 Vibrational signature of the chimney . . . . . . . . . . . . . . 42

4.3 Targets without no concealed vibrating sources . . . . . . . . . . . 43

4.4 Vibrational signature of the rocking quad-corner reflector . . . . . . 43

4.5 Vibrational signature of the sliding quad-corner reflector . . . . . . 44

4.6 Layout of the targets on the ground scene . . . . . . . . . . . 45

4.7 SAR image of the ground scene at 4-in resolution image . . . . . . . 45

4.8 Estimated vibrational signature of the top-hat chimney reflector . . 46

4.9 Estimated vibrational signature of the rocking quad-corner reflector 47

4.10 Estimated vibrational signature of the sliding quad-corner reflector 48

B.1 Subspace methods and analytical expressions . . . . . . . . . . 56 


\section{List of Tables}

1.1 Multiplicities of DFT eigenvalues . . . . . . . . . . . . 6

4.1 SAR system parameters for the 4-in resolution data. . . . . . . . . . 44

4.2 Parameters employed in the DFRFT-based vibrometry technique. . 46

4.3 Parameters employed in the DFRFT-based vibrometry technique. . 49 


\section{Abbreviations}

2D

AWGN

BW

CDFRFT

CDFT

$c_{r}$

DFT

DFRFT

DTFT

FFT

FIR

FRFT

G-H

IIR

MLE

MSE

QMFD

QMOD
2-Dimensional

Additive Gaussian White Noise

Bandwidth

Centered Discrete Fractional Fourier Transform

Centered Discrete Fourier Transform

Chirp Rate

Discrete Fourier Transform

Discrete Fractional Fourier Transform

Discrete Time Fourier Transform

Fast Fourier Transform

Finite Impulse Response

Fractional Fourier Transform

Gauss-Hermite

Infinite Impulse Response

Multiple Angle-Centered Discrete Fractional Fourier Transform Maximum Likelihood Estimators

Mean Squared Error

Quantum Mechanics in Finite Dimensions

Modified QMFD 
SAR

SLAR

SNR

SCR

$w_{c}$
Synthetic Aperture Radar

Side Looking Airborne Radar

Signal-to-Noise Ratio

Signal-to-Clutter Ratio

Central Frequency 


\section{Chapter 1}

\section{Introduction}

\subsection{Overview}

The estimation of the frequency and chirp rate of a chirp signal in a noisy environment is a fundamental and well-studied problem in signal processing and communications. Its numerous applications include carrier recovery in a communication system [1], determination of the object position and speed in radar and sonar systems[2], estimation of the heart rate of a fetus in biomedicine [3], and many other. Regardless of the application, poor estimation can lead to inaccurate results. For example, in communication systems, with a poor carrier frequency estimate, the down-converter may not be able to properly demodulate the passband signal to baseband [1]. In radar applications, a poor estimate of the chirp rate may cause the system to fail in accurately calculating the position and velocity of the target [4].

A variety of approaches to the center frequency and chirp rate estimation problem, distinguished primarily by their estimation accuracy, and computational complexity, have been developed. One of the approaches is based on improving the Santhanam-Peacock empirical estimation [5] algorithm namely the discrete fractional Fourier transform (DFRFT) approach. We specifically look at the distortion issues associated with the discretization and provide an operator with improved 
estimation via use of suitable windowing of the eigenvalue spectrum. The issue with this estimation is its computational complexity as well as its empirical nature.

Another class of approaches are based on FFT due to its connection with the maximum likelihood estimation (MLE) of frequency and thus chirp rate. The MLE has very high accuracy because it achieves the Cramer-Rao lower bound (CRLB), which is the minimum possible error for the estimator, over a range of signal-to-noise (SNR) values. In this thesis, we improvise the prior work by Ishwor [19] to develop analytical expression for the parameter estimates instead of the empirical one. But since we are using centered DFT (CDFT), for even $N$, there is no coefficient for zero frequency while computing it. This problem is solved by shifting the signal to be transformed by a frequency of $\pm \pi / N$ which we call "half-sample" shift. This shift produces the DC component coefficient from the CDFT when $N$ is even. We incorporated this shift in the estimator itself in order to compensate for the shifting of the signal.

Another class of approaches address the shortcomings of the previous estimator and is refined by zero padding, spectral peak interpolation and chirp-z-transform based zooming techniques. Several interpolation techniques, zooming factors and different zero padding length and their effect are studied and compared in terms of mean squared error (MSE) of the parameter estimates with respect to CRLB. Finally, we show the combination of these refinements along with the analytical expressions significantly improve the MSE of the estimates.

We finally apply the improved chirp parameter estimation approach to the problem of estimating the chirp rate and instantaneous acceleration of a vibrating target. This approach applied to synthetic aperture radar (SAR) data provides for acceleration estimation very close to the accelerometer information of these targets. 


\subsection{Fractional Fourier Transform}

The fractional Fourier transform (FRFT) is a family of linear transformations which generalize the Fourier Transform. It can be thought of as the Fourier transform to the nth power, where n need not to be an integer. Thus, it can transform to any intermediate domain between time and frequency. The FRFT depends on a parameter $\alpha$ and can be interpreted as a rotation by an angle $\alpha$ in the timefrequency plane. So, an FRFT with $\alpha=\pi / 2$ corresponds to the classical Fourier transform, and an FRFT with $\alpha=0$ corresponds to the identity operator. It was introduced a numbers of years ago in the mathematics literature [6] but appears to have remained largely unknown to the signal processing community, to which it may, be potentially useful. Now, it has been shown that there is a close relationship between the linear chirp signal and the continuous FRFT as the FRFT of a signal can be interpreted as a decomposition in terms of chirps.

For any real angle $\alpha$, the $\alpha$-angle FRFT of a function $f(x)$ is denoted by $\mathbf{F}_{\alpha}(u)$ and defined by,

$$
\mathbf{F}_{\alpha}(u)=\sqrt{\frac{1-j \cot (\alpha)}{2 \pi}} \exp \left(\frac{j \pi \cot (\alpha) u^{2}}{2}\right) \int_{-\infty}^{\infty} I_{\alpha} f(x) d x
$$

where $I_{\alpha}=\exp \left(-j 2 \pi\left(\csc (\alpha) u x-\frac{\cot (\alpha)}{2} x^{2}\right)\right)$.

This is why when $\alpha=\frac{\pi}{2}$, this precisely becomes the definition of the continuous Fourier transform, and for $\alpha=-\frac{\pi}{2}$, it is the definition of the inverse continuous Fourier transform.

In other words, the FRFT is an integral transform

$$
\mathbf{F}_{\alpha}(u)=\int \mathbf{K}_{\alpha}(u, x) f(x) d x
$$


where $\mathbf{K}_{\alpha}(u, x)$ is the transformation kernel defined as,

$$
\mathbf{K}_{\alpha}(u, x)=\left\{\begin{array}{ll}
\sqrt{\frac{1-\jmath \cot (\alpha)}{2 \pi}} \exp \left(\jmath A_{\alpha}\right), & \text { if } \alpha \text { is not a multiple of } \pi \\
\delta(u-x), & \text { if } \alpha \text { is a multiple of } 2 \pi \\
\delta(u+x), & \text { if } \alpha+\pi \text { is a multiple of } 2 \pi
\end{array},\right.
$$

where $A_{\alpha}=\left(\frac{x^{2}+y^{2}}{2} \cot (\alpha)-u x \csc (\alpha)\right)$. This kernel is continuous in $\alpha$, i.e. in multiples of

$$
\lim _{\alpha \rightarrow n \pi} \mathbf{K}_{\alpha}=\mathbf{K}_{n \pi}
$$

Thus, substituting Eq. (1.3) in Eq. (1.2), we get,

$$
\mathbf{F}_{\alpha}(u)=\left\{\begin{array}{ll}
\sqrt{\frac{1-\jmath \cot (\alpha)}{2 \pi}} \exp \left(\jmath\left(\frac{u^{2}}{2} \cot (\alpha)\right)\right) \int_{-\infty}^{\infty} f(x) \exp \left(\jmath B_{\alpha}\right) d x, & \text { if } \alpha=k \pi \\
f(x), & \text { if } \alpha=2 k \pi \\
f(-x), & \text { if } \alpha+\pi=2 k \pi
\end{array},\right.
$$

where $B_{\alpha}=\left(\frac{x^{2}}{2} \cot (\alpha)+u x \csc (\alpha)\right)$ and $k=$ integer.

\subsection{Discrete Fractional Fourier Transform}

The DFRFT generalizes the discrete Fourier transform (DFT) in the same sense that the continuous FRFT generalizes the continuous Fourier transform. As the DFT is defined as a linear operator that can be expressed via a matrix vector multiplication, analogously, the definition of DFRFT is based on a particular set of eigenvectors of the DFT matrix or well-known eigenvalue-eigenvector decomposition of a matrix. The DFRFT can be generally put in the form as [7],

$$
\mathbf{A}_{\alpha}(x)=\mathbf{W}^{\frac{2 \alpha}{\pi}(x)}=\mathbf{V} \Lambda^{\frac{2 \alpha}{\pi}} \mathbf{V}^{-1} x
$$

where $\mathbf{W}$ is a DFT matrix, $\mathbf{V}$ is DFT eigenvectors and $\Lambda$ is a diagonal matrix of DFT eigenvalues. 
In the case of the regular DFT, these eigenvectors are linearly independent and orthonormal. So, the inverse of these eigenvector in this case can be replaced by its hermitian, i.e. DFT matrix $\mathbf{W}$ can be written as,

$$
\mathbf{W}=\mathbf{V} \Lambda \mathbf{V}^{H}
$$

Substituting this information in Eq. (1.6), we get,

$$
\mathbf{A}_{\alpha}(x)=\mathbf{W}^{\frac{2 \alpha}{\pi}(x)}=\mathbf{V} \Lambda^{\frac{2 \alpha}{\pi}} \mathbf{V}^{H} x
$$

Now, if we evaluate Eq. (1.8) for different value of $\alpha=0, \frac{\pi}{2}$, and $2 \pi$ we obtain,

$$
\mathbf{A}_{2 \pi}=\mathbf{I}, \mathbf{A}_{\frac{\pi}{2}}=\mathbf{W}, \mathbf{A}_{\pi}=\mathbf{W}^{2}
$$

\subsection{Discrete Rotational Fourier Transform}

A first discussion on DFRFT was introduced by Santhanam and McClellan in [7]. This transform was called rotational to show the fact that it involved rotation in time-frequency plane.

The formulation of the rotational form of the DFRFT is simple. It is a linear combination of integer powers of the DFT matrix, the resulting transform does not quite resemble the continuous version. The expression given in [7] is based on a grouping of eigenvalues that results in eigenvectors of the DFT that are linearly independent but form a non-orthogonal basis. The DFT matrix has only four eigenvalues $(1,-1, j,-j)$ regardless of its size for $N \geq 4$ [8]. Therefore we have repeated eigenvalues for $\mathrm{N}>4$. This repetitive nature of the eigenvalues produces several problems. One of the problems is that the DFT has several sets of orthogonal eigenvectors. For a matrix to have a complete set of orthogonal eigenvectors, unique eigenvalues [9] is a must but since this is not the case for DFT, there are multiple sets of orthogonal eigenvectors.

Also, any linear combination of the DFT eigenvectors with the same eigenvalue 


\begin{tabular}{|c|c|c|c|c|}
\hline $\mathrm{N}$ & 1 & $-\mathrm{j}$ & -1 & $\mathrm{j}$ \\
\hline $4 \mathrm{~m}$ & $\mathrm{~m}+1$ & $\mathrm{~m}$ & $\mathrm{~m}$ & $\mathrm{~m}-1$ \\
\hline $4 \mathrm{~m}+1$ & $\mathrm{~m}+1$ & $\mathrm{~m}$ & $\mathrm{~m}$ & $\mathrm{~m}$ \\
\hline $4 \mathrm{~m}+2$ & $\mathrm{~m}+1$ & $\mathrm{~m}$ & $\mathrm{~m}+1$ & $\mathrm{~m}$ \\
\hline $4 \mathrm{~m}+3$ & $\mathrm{~m}+1$ & $\mathrm{~m}+1$ & $\mathrm{~m}+1$ & $\mathrm{~m}$ \\
\hline
\end{tabular}

Table 1.1: Multiplicities of DFT eigenvalues [7]

are DFT eigenvectors. So, there is ambiguity problem in deciding the eigenvectors of the DFT. The multiplicities of the eigenvalues of the DFT are shown in Table 1.1. From this table, we can see that the DFT has a non-uniform eigenvalue distribution when $\mathrm{N}$ is a multiple of four.

\subsection{Cramer-Rao Lower Bound}

To evaluate the performance of the estimators, it is valuable to compare the associated MSE with respect to the theoretical bounds by Cramer and Rao. This bound has been calculated before chirps [10], but there are different forms of the chirp function given in different papers [5]. Here is a summary for the specific form of the lower bound used in this thesis [5].

The components of the Fisher information matrix for any signal in complex additive White Gaussian noise (AWGN) is,

$$
J_{i j}=\frac{2}{\sigma^{2}} \sum_{n=0}^{N-1}\left(\frac{\partial \mu_{n}}{\partial \Theta_{i}} \frac{\partial \mu_{n}}{\partial \Theta_{j}}+\frac{\partial \nu_{n}}{\partial \Theta_{i}} \frac{\partial \nu_{n}}{\partial \Theta_{j}}\right)
$$

where $\mu_{n}=\operatorname{real}(f[n])$ and $\nu_{n}=\operatorname{imag}(f[n])$, are the expected values of real and imaginary components of the signal. For multicomponent case $\Theta=\left[\theta_{1}, \theta_{2}, \ldots, \theta_{P}\right]^{T}$ and $J$ will have the form:

$$
\left[\begin{array}{ccccc}
J_{11} & P_{12} & J_{13} & \ldots & J_{1 P} \\
J_{21} & P_{22} & J_{23} & \ldots & J_{2 P} \\
\vdots & \vdots & \vdots & \ddots & \vdots \\
J_{P 1} & J_{P 2} & J_{P 3} & \ldots & J_{P P}
\end{array}\right]
$$


composed of the block matrices

$$
J_{i j}=\frac{2}{\sigma^{2}} \sum_{n=0}^{N-1}\left[\begin{array}{cccc}
c_{i j}[n] & A_{j} s_{i j}[n] m^{2} & A_{j} s_{i j}[n] n & A_{j} s_{i j}[n] \\
-A_{j} s_{i j}[n] m^{2} & A_{i} A_{j} c_{i j}[n] m^{4} & A_{i} A_{j} c_{i j}[n] m^{2} n & A_{i} A_{j} c_{i j}[n] m^{2} \\
-A_{i} s_{i j}[n] n & A_{i} A_{j} c_{i j}[n] m^{2} n & A_{i} A_{j} c_{i j}[n] n^{2} & A_{i} A_{j} c_{i j}[n] n \\
-A_{i} s_{i j}[n] & A_{i} A_{j} c_{i j}[n] m^{2} & A_{i} A_{j} c_{i j}[n] n & A_{i} A_{j} c_{i j}[n]
\end{array}\right]
$$

where

$$
\begin{aligned}
& c_{i j}[n]=\cos \left(\Phi_{i}[n]-\Phi_{j}[n]\right) \\
& s_{i j}[n]=\sin \left(\Phi_{i}[n]-\Phi_{j}[n]\right) \\
& \Phi_{i}[n]=\alpha_{i} m^{2}+\omega_{i} n+\phi_{i}
\end{aligned}
$$

For a single chirp, the inverse of this matrix has a closed form, and the Cramer-Rao lower bound can be computed as [5]

$$
\begin{aligned}
& \operatorname{var}\{\hat{\alpha}\} \geq\left(\frac{\sigma}{A}\right)^{2} \frac{90}{N\left(N^{2}-1\right)\left(N^{2}-2\right)} \\
& \operatorname{var}\{\hat{\omega}\} \geq\left(\frac{\sigma}{A}\right)^{2} \frac{6}{N\left(N^{2}-1\right)}
\end{aligned}
$$

For the multicomponent case, there is no closed form. All the results presented for the single component case hold approximately for each of the components, as long as the components are spectrally well separated [11] i.e. if the chirps are well-separated in frequency, the above bounds hold true.

\subsection{Organization of the thesis}

In this thesis, we look to quantify the accuracy of the refined Santhanam-Peacock chirp parameter estimates. We study the different methods and present a improved accuracy estimator. This thesis is organized as follows:

Chapter 2 is dedicated to minimizing the errors during the discretization of the continuous FRFT. The eigenvectors, and the basis functions with some properties 
are explained. In this chapter, we also briefly explain the empirical mapping and invertibility percentage which shows the improvements in the estimates. We plot the valid mapping region for peak-to-parameter mapping. Finally, we compare the parameter estimates in terms of MSE over a range of SNR.

In Chapter 3, we introduce analytical expressions for the chirp parameter estimators based on the CDFT and apply different refinements to it such as zero padding, spectral peak interpolation and CZT zoom-in [12]. A brief introduction to all the techniques is presented and modifications to the analytical expressions based on these refinements are also discussed. We compute the MSE over a range of SNR values for all the techniques and finally combine them for better estimates for a given signal length.

Chapter 4 presents the application of these new analytical expressions and refinements to the problem of estimating vibrating target parameters in a SAR- based vibrometry application. We study three different types of vibrating targets and their unique vibration signatures. We compare the results from our algorithm to the ground truth measured from the accelerometer attached to the targets.

Finally, in Chapter 5, we conclude our investigation and discuss topics for future research. 


\section{Chapter 2}

\section{Effects of Windowing}

In this chapter, we first look at the Vargas-Santhanam [20] empirical estimation method which was refined by Santhanam-Peacock by the use of subspace decomposition [5]. We then focus on the distortion issues, that arise in the transition of continuous FRFT to DFRFT and incorporate windowing effects into the QMFD approach [13][17]. We further investigate extensions of the QMFD approach [13][18][17] in light of these distortion sources, that result in a commuting matrix and associated eigenvectors with a reduced degree of distortion. We further show through simulation results, that a suitable choice of window applied to the eigenvalue sequence of the discrete operator results in significant improvement of the invertibility of the underlying peak to parameter mapping and thus, to the associated MSE of chirp parameter estimates.

\subsection{Empirical Estimation \& Subspace Decompo- sition}

The DFRFT technique shows promise for multicomponent chirp parameter estimation as it generates peaks for each chirp whose location in the $2 \mathrm{D}$ transform plane corresponding to the specific chirp rate and center frequency. The peak-toparameter mapping was first investigated in [20] in a closed-form empirical ap- 
proximation of the relationship, however this approximation had significant error in parameter estimation.

Subspace decomposition techniques in conjunction with the DFRFT provides more robust and accurate estimation in the presence of noise [37]. This method, however, causes the peak to be suppressed resulting in lower performance of the subspace decomposition. Santhanam-Peacock [5] investigated using different p-norms for the projection to better accentuate the peaks, and put forward a cross-hairs method, where subspace decomposition is performed on the thin slices centered around the peak. In this method, horizontal and vertical projections at the peak location is calculated using a p-norm

$$
\begin{gathered}
x_{\alpha}=\mathrm{FFT}^{-1}\|\mathbf{X}\|_{p}^{\text {row }}=\mathrm{FFT}^{-1}\left(\sum_{k=K_{0}}^{K_{f}}\left|X_{k}[\bullet]\right|^{p}\right)^{\frac{1}{p}} \\
x_{\omega}=\mathrm{FFT}^{-1}\|\mathbf{X}\|_{p}^{c o l}=\mathrm{FFT}^{-1}\left(\sum_{k=R_{0}}^{R_{f}}\left|X_{\bullet}[r]\right|^{p}\right)^{\frac{1}{p}}
\end{gathered}
$$

The peaks in the projection result in strong frequency content in the signals $x_{\alpha}$ and $x_{\omega}$. This turns the chirp parameter estimation into two separable frequency estimation problems, which can be easily solved using subspace decomposition. The use of the 3-norm has a higher noise-floor at high SNR. but less at low SNR. So, it was selected as a good compromise and for further evaluation.

The subspace decomposition process begins by performing the eigenvalue decomposition on the estimated covariance matrices $\mathbf{R}_{\alpha}$ and $\mathbf{R}_{\omega}$ of size $(C+M) * N$, where $C$ is the number of chirps in the signal. The eigenvectors corresponding to the $C$ largest eigenvalues were selected to form signal subspace while $M$ eigenvectors are used to form noise subspace. Next, pseudo-spectra is calculated using min-norm method as

$$
P_{\text {min-norm }}=\frac{1}{\left|\operatorname{FFT}\left(\mathbf{V V} \mathbf{V}_{1}^{T}\right)\right|},
$$

where $\mathbf{V}$ is the eigenvector matrix and $\mathbf{V}_{1}^{T}$ denotes a column vector containing the first element of each eigenvector. Finally, this pseudo-spectra is searched for largest $C$ peaks. 


\subsection{Distortion Sources}

As mentioned in 1.3, the kernel of the continuous-time FRFT is

$$
K_{\alpha}(t, u)=\sqrt{\frac{1-j \cot \alpha}{2 \pi}} \exp \left(j\left(t^{2}+u^{2}\right) \cot \alpha / 2-j t u \csc \alpha\right)
$$

The corresponding Mehler's expansion for the chirped kernel is

$$
K_{\alpha}(t, u)=\sum_{k=0}^{\infty} \exp (-j k \alpha) h_{k}(t) h_{k}(u)
$$

where $h_{k}(t)$ denotes the $k$-th Gauss-Hermite $(\mathrm{G}-\mathrm{H})$ functions. When a chirp signal with center frequency $\omega$ and chirp rate $c_{r}$ for a specific angle $\alpha_{o}$ is used, this kernel produces a Dirac-impulse for the FRFT

$$
F_{\alpha_{o}}(u)=\exp \left(j \frac{\cot \alpha_{o}}{2} u^{2}\right) \sqrt{\frac{1-j \cot \alpha_{o}}{2 \pi}} 2 \pi \delta\left(\omega_{o}-u \csc \alpha_{o}\right)
$$

where $\alpha_{o}=\cot ^{-1}\left(-2 c_{r}\right)$. This chirped kernel is not band-limited and causes distortion in the discrete versions of the FRFT. Existing commuting matrix methods for computing the discrete version of the FRFT (DFRFT) [13][14] [15] use a eigenvalue decomposition of the form

$$
\mathbf{A}_{\alpha}(x)=\mathbf{V} \Lambda^{\frac{2 \alpha}{\pi}} \mathbf{V}^{T} x=\sum_{k=0}^{N-1} \exp (-j k \alpha) v_{k} v_{k}^{T} x
$$

where $\mathbf{V}$ is a fully orthogonal basis of DFT or CDFT eigenvectors, obtained from a commuting matrix, that serves as discrete counterparts of the $\mathrm{G}-\mathrm{H}$ functions.

\subsection{Sources of Discretization Errors}

From Eq. (2.5) and Eq. (2.7), we can observe that discretizing the continuous FRFT, there are following phenomena happening [17]

1. Truncation or windowing of the IIR eigenvalue sequence of the G-H operator 
with a rectangular window of the duration $N$ samples

$$
\lambda_{\omega}[k]=\exp (-j k \alpha) \omega[k]
$$

where $\omega[k]$ is the $N$-point boxcar window [16]. This is analogous to the window based FIR filter design technique, where a IIR impulse response is approximated with an FIR windowed equivalent or spectral analysis using the DFT. This will result in spectral distortion of the eigenvalue sequence

$$
\Lambda_{\omega}\left(e^{j w}\right)=W\left(e^{j(\omega-\alpha)}\right)
$$

where $W\left(e^{j \omega}\right)$ denotes the discrete time Fourier transform (DTFT) of the window function [16] used. This produces the narrowest main lobe but has the smallest main-lobe to side-lobe spectral amplitude ratio which produces more side-lobes in the DTFT of the windowed eigenvalue sequence. G$\mathrm{H}$ operator discretization methods discussed so far do not accommodate windowing effects.

2. Discretization of the G-H functions to yield DFT/CDFT eigenvectors which results in spectral aliasing in the eigenvectors corresponding to the higher G-H modes with frequency content at the edges of $|\omega|<\pi$,

$$
V_{k}\left(e^{j \omega}\right)=\frac{1}{T_{s}} \sum_{k=-\infty}^{\infty} H_{k}\left(\frac{\omega-2 k \pi}{T_{s}}\right), \quad|\omega|<\pi
$$

where $T_{s}$ is the sampling period associated with the discretization of the G-H functions.

Several commuting matrix approaches towards presenting the unitary basis of DFT/CDFT eigenvectors have been studied [13][14] [15] showing that they are discrete versions of the G-H operator. 


\subsection{QMFD Approach : Diagonal Q and Quasi- Toeplitz Matrices}

First, we focus our attention on the QMFD approach in [13] [18]

$$
\begin{aligned}
\mathbf{Q} & =\sqrt{\frac{2 \pi}{N}} \operatorname{diag}(-m, \ldots, m) \\
\mathbf{P} & =\mathbf{W} \mathbf{Q} \mathbf{W}^{H} \\
\mathbf{T} & =\mathbf{P}^{2}+\mathbf{Q}^{2},
\end{aligned}
$$

where $\mathbf{Q}$ and $\mathbf{P}$ denote the finite dimensional position and momentum operators and $\mathbf{W}$ denotes the centered version of the DFT

$$
\mathbf{W}_{r s}=\frac{1}{\sqrt{N}} \exp \left(-j \frac{2 \pi}{N}(r-m)(s-m)\right), \quad 0 \leq r, s \leq N-1
$$

with $m=(N-1) / 2$. As shown in Eq. (2.8), for the matrix $\mathbf{T}$ to commute with either version of the DFT or CDFT, the matrix $\mathbf{W}^{2}$ needs to be $\mathbf{W}^{2}$ centrosymmetric

$$
\mathbf{W}^{2} \mathbf{Q}^{2} \mathbf{W}^{2}=\mathbf{Q}^{2}
$$

If we require the commutator $\mathbf{C}=[\mathbf{Q}, \mathbf{P}]$ to commute with the DFT, we would require the matrix $\mathbf{Q}$ to be $\mathbf{W}^{2}$ anti-symmetric [13]

$$
\mathbf{W}^{2} \mathbf{Q} \mathbf{W}^{2}=-\mathbf{Q}
$$

Here, we specifically focus on the elements of the matrix $\mathbf{P}$

$$
\mathbf{P}_{r s}=\sum_{l=0}^{N-1} \sum_{m=0}^{N-1} \mathbf{W}_{r l} \mathbf{Q}_{l m} \mathbf{W}_{m s}^{*}
$$

Substituting the diagonal form of $\mathbf{Q}$ into this expression gives

$$
\mathbf{P}_{r s}=\sqrt{\frac{2 \pi}{N}} \sum_{l=0}^{N-1} \exp \left(-j \frac{2 \pi}{N}(l-m)(r-s)\right) .
$$


Specifically the matrix $\mathbf{Q}$ is non-diagonal, is purely imaginary because its elements are the DFT of an odd function. The matrix $\mathbf{Q}$ is also Toeplitz since the matrix elements depend only on $(r-s)$. In a similar fashion, we can evaluate the matrix elements of the commuting matrix $\mathbf{T}$ via

$$
\mathbf{T}_{r s}=\frac{2 \pi}{N} \begin{cases}\sum_{l=0}^{N-1}(l-m)^{2} \exp \left(-j \frac{2 \pi}{N}(l-m)(r-s)\right), & \text { when } r \neq s \\ (r-m)^{2}+\sum_{l=0}^{N-1}(l-m)^{2}, & \text { when } r=s\end{cases}
$$

The following symmetries can be inferred from the matrix elements

1. For the diagonal form of the $\mathbf{Q}$ matrix with either the DFT or CDFT, the underlying commuting matrix has almost-Toeplitz symmetry.

2. Only main diagonal elements are different and follow a square law in accordance with the $(r-m)^{2}$ or the $(r-m-1)^{2}$ terms. Non-Toeplitz behavior of the commuting matrix is a consequence of non-Toeplitz behavior of the matrix $\mathbf{Q}$.

3. The commuting matrix will also have $\mathbf{J}$-symmetry about $r=m$ along the diagonal for the CDFT and $\mathbf{W}$-symmetry about $r=m+1$ along the diagonal of DFT.

4. The commuting matrix is further positive semi-definite.

This motivates the equivalence of the commuting matrix $\mathbf{T}$ to the auto-correlation matrix of a weakly non-stationary time-series with elements

$$
\begin{array}{ll}
\frac{2 \pi}{N} \sum_{l=0}^{N-1}(l-m-1)^{2} \exp \left(-j \frac{2 \pi}{N}(l-m)(r-s)\right), & \text { when } r \neq s \\
\frac{2 \pi}{N}(r-m-1)^{2}+\frac{2 \pi}{N} \sum_{l=0}^{N-1}(l-m-1)^{2} & , \text { when } r=s
\end{array}
$$

This weakly non-stationary time-series, autocorrelation viewpoint, exposes the aliasing in the almost-Toeplitz framework, since the quadratic power spectral term is not band-limited. The conclusion derived from this viewpoint is that the QMFD 
discretization of the non band-limited G-H operator will result in distortion [17]. This distortion manifests as aliasing in the eigenvectors.

The time-series viewpoint also describes the windowing effects on the elements of the quasi-Toeplitz commuting matrix. Specifically the elements of the $\mathbf{Q}^{2}$ matrix are windowed with a rectangular window of duration $N$ samples

$$
\mathbf{Q}_{\omega}^{2}=\Lambda_{\omega} \mathbf{Q}^{2}
$$

where $\Lambda_{\omega}$ is a diagonal matrix with the window samples along the diagonal. We can affect the eigenvalue spectrum of the commuting matrix by choosing an appropriate window as depicted in Fig. (2.1), where we use a Kaiser window of duration $N=256$ and parameter $\beta=1.2$. This window is chosen over others due to the degree of freedom that the parameter $\beta$ affords in the main-lobe to side-lobe trade-off [17]. This has the effect of smoothing the fluctuations in the eigenvalue spectrum at the tail end as evident in Fig. (2.2) and expanding the region of the invertibility of the peak to parameter mapping [19].

In order to further reduce the distortions resulting from the truncation/windowing of the eigenvalue sequence, we now consider the non-diagonal $\mathbf{Q}$ case where the DFT commuting matrix is forced to possess a linear eigenvalue spectrum.

\subsection{QMFD Approach: Non-Diagonal Case}

As described in [18], the QMFD method needs to be modified so that the equations of motion are satisfied in the DFT/CDFT basis. This is because the $\mathbf{Q}$ and $\mathbf{P}$ tridiagonal basis corresponds to a diagonal number operator and a diagonal DFT operator. To obtain the number operator in the DFT/CDFT basis, we perform similarity transformation via the eigenvectors of the DFT/CDFT obtained from the previous section. Specifically, the $\mathbf{Q}$ and $\mathbf{P}$ tridiagonal matrices in the $\mathrm{N}$ - 


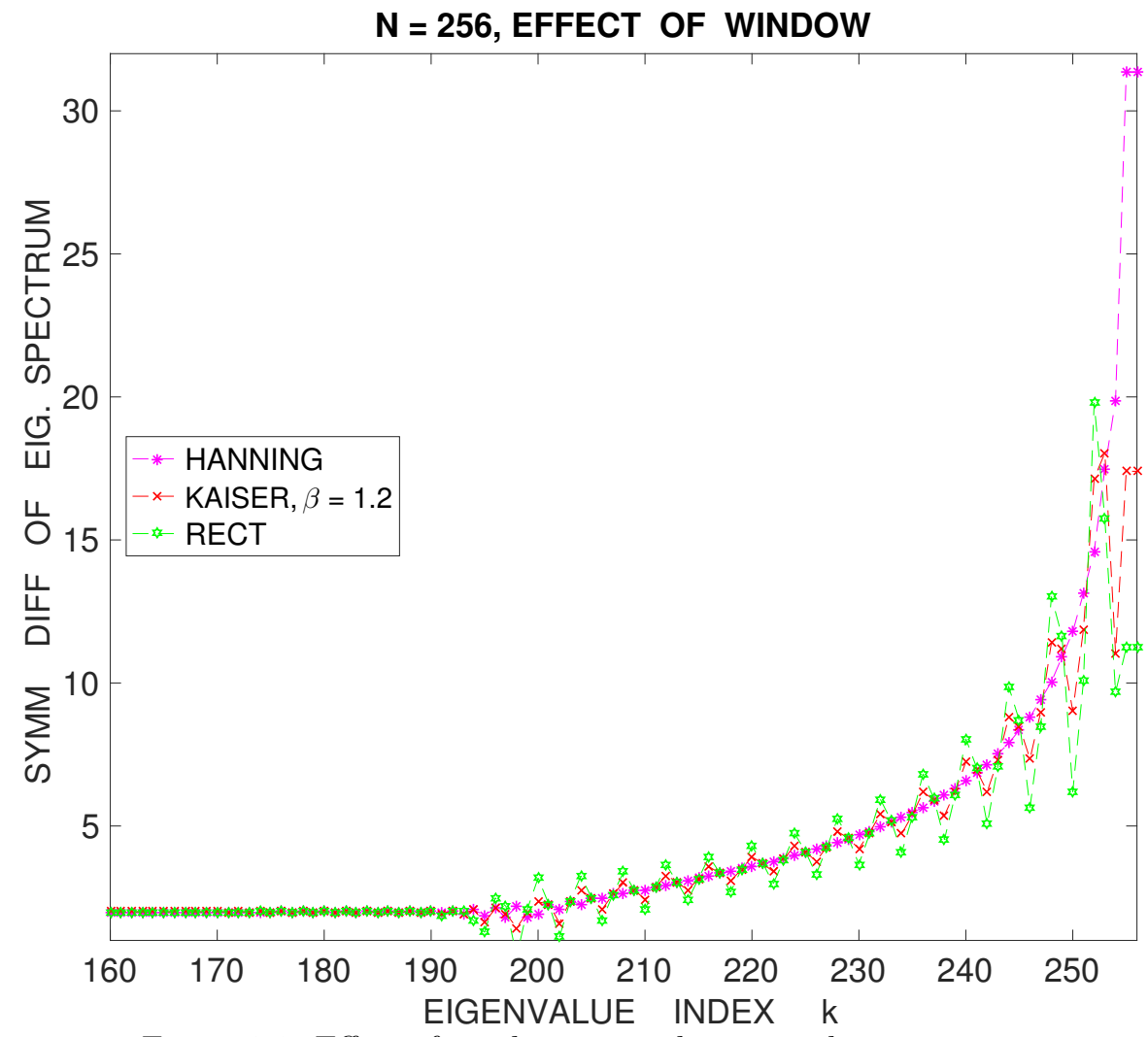

Figure 2.1: Effect of windowing on the eigenvalue spectrum.

diagonal basis or the DFT diagonal basis [17] are

$$
\begin{gathered}
\mathbf{Q}_{o}=\frac{1}{\sqrt{2}}\left(\begin{array}{ccccc}
0 & 1 & 0 & 0 & \ldots \\
1 & 0 & \sqrt{2} & 0 & \ldots \\
0 & \sqrt{2} & 0 & \sqrt{3} & \ldots \\
0 & 0 & \sqrt{3} & 0 & \sqrt{4} \ldots \\
\vdots & \vdots & \vdots & \vdots & \ldots
\end{array}\right) \\
\mathbf{P}_{o}=\frac{j}{\sqrt{2}}\left(\begin{array}{cccccc}
0 & 1 & 0 & 0 & \ldots \\
-1 & 0 & \sqrt{2} & 0 & \ldots \\
0 & -\sqrt{2} & 0 & \sqrt{3} & \ldots \\
0 & 0 & -\sqrt{3} & 0 & \sqrt{4} \ldots \\
\vdots & \vdots & \vdots & \vdots & \ldots
\end{array}\right)
\end{gathered}
$$

The number operator in the tridiagonal representation is just the diagonal matrix

$$
\mathbf{N}_{o}=\operatorname{diag}(1,3,5,7, \ldots, 2 N-1)
$$




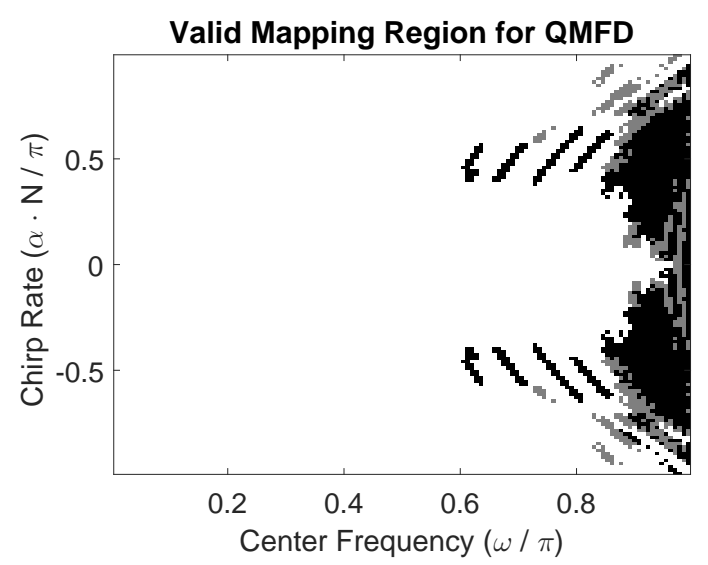

(a)

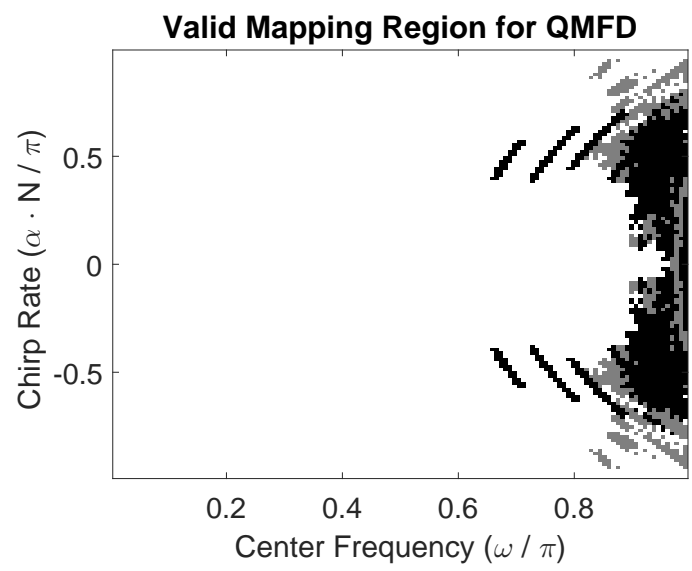

(b)

Figure 2.2: Peak to parameter mappings for $N=256$ : (a) quasi-Toeplitz framework, and (b) non-diagonal $\mathbf{Q}$ formulation. The invertibility percentage of the mappings corresponding to the two operators are $84.55 \%$ and $85.33 \%$ respectively.

These quantities in the DFT basis are obtained via similarity transformation using the DFT eigenvectors $\mathbf{V}$ obtained from the previous section

$$
\mathbf{Q}_{\text {new }}=\mathbf{V Q}_{o} \mathbf{V}^{H} \text { and } \mathbf{P}_{\text {new }}=\mathbf{V} \mathbf{P}_{o} \mathbf{V}^{H}
$$

The corresponding number operator in the DFT basis is:

$$
\mathbf{T}=\mathbf{P}_{\text {new }}^{H} \mathbf{P}_{\text {new }}+\mathbf{Q}_{\text {new }}^{H} \mathbf{Q}_{\text {new }}=\mathbf{P}_{\text {new }}^{2}+\mathbf{Q}_{\text {new }}^{2}=\mathbf{V N}_{o} \mathbf{V}^{H}
$$

where $\mathbf{V}$ are the orthogonal DFT eigenvectors obtained via the previous section. This transformed number operator by construction has a odd integer eigenvalue spectrum, and its eigenvectors are the DFT eigenvectors. This matrix therefore commutes with the appropriate DFT:

$$
\mathbf{T W}=\mathbf{W} \mathbf{T} \text { or }[\mathbf{W}, \mathbf{T}]=\mathbf{0} .
$$

The transformed number operator is however, not almost-Toeplitz as in the previous section but a $\mathbf{W}^{2}$-symmetric matrix corresponding to a non-diagonal $\mathbf{Q}_{\text {new }}$ matrix:

$$
\mathbf{W}^{2} \mathbf{T} \mathbf{W}^{2}=\mathbf{T} \mathbf{W}^{4}=\mathbf{T}
$$


where we have used the observation that $[\mathbf{W}, \mathbf{T}]=\mathbf{0}$. Since the new number operator was constructed from the eigenvectors from the previous almost-Toeplitz framework, they carry with them the distortion due to truncation and aliasing. However, the distortion is reduced because the eigenvalue spectrum is closer to that of the G-H operator than what was obtained in the previous framework, thereby reducing one of the sources of distortion [17]. We are specifying the eigenvalue sequence of the commuting matrix to be the truncated odd numbered spectrum. We can further reduce the effects of windowing of the eigenvalue sequence by choosing the window so that the eigenvalue sequence is:

$$
\lambda_{w}[k]=(2 k+1) w[k], \quad 0 \leq k \leq N-1
$$

where $w[k]$ is an appropriately chosen window [16] that minimizes the effects of eigenvalue truncation.

Fig. (2.2) compares the peak to parameter mapping underlying both the diagonal Q approach and the non-Toeplitz framework for $N=256$ using the Kaiser window. There is slight improvement visually as well as in terms of invertibility of the mapping from 84.55 percent to 85.33 percent. This improvement is due to the fact that non-diagonal $\mathbf{Q}$ case minimizes the effects of eigenvalue truncation[17].

Fig. (2.3)(a) depicts the DFRFT spectra for a chirp signal for different values of the Kaiser window parameter $\beta$. For $\beta=15$ in non-diagonal $\mathbf{Q}$ case, we can observe that there is significant distortion of the peaks arising from truncation. As can be seen specific values of the $\beta$ parameter result in steeper slopes on the peaks of the underlying DFRFT spectrum. From Fig. (2.3)(b,c) we observe that the slopes of the spectral peaks are much steeper for $\beta=1.2$ than for $\beta=0.001$. Fig. (2.3)(d) depicts the eigenvalue spectrum corresponding to the different values of the Kaiser window parameter [17].

Fig. (2.4) depicts the percentage invertibility associated with the $\mathbf{Q}$-windowing approach, the eigenvalue windowing approach, and the combined windowing approach using a Kaiser window with parameter $\beta=1.2$. We can see that the combined windowing approach improves the invertibility of the peak to parameter 


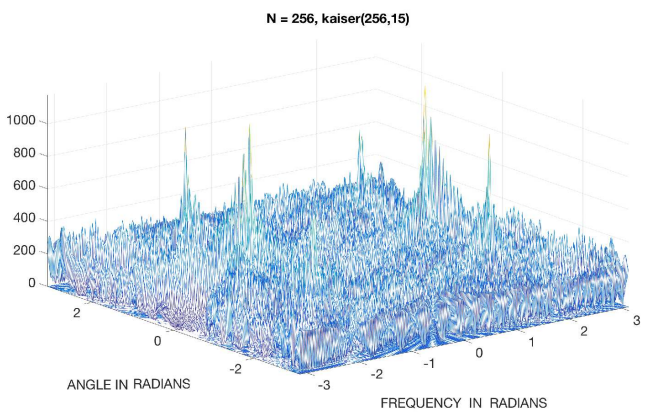

(a)

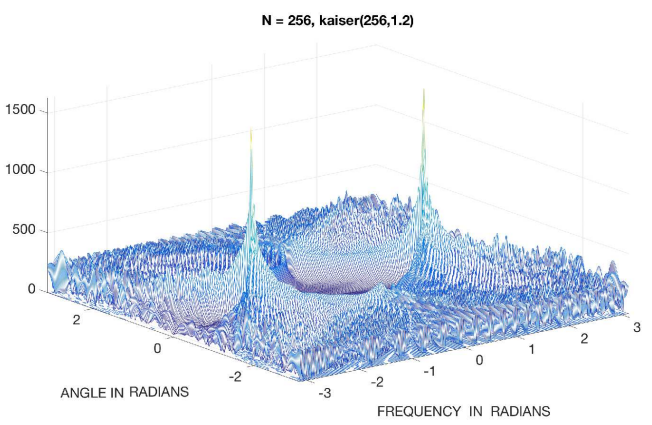

(c)

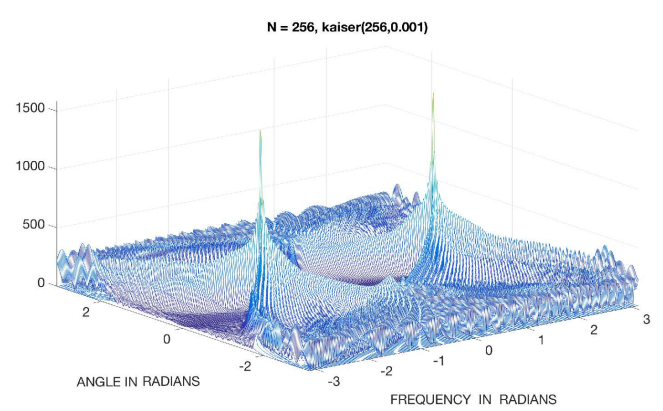

(b)

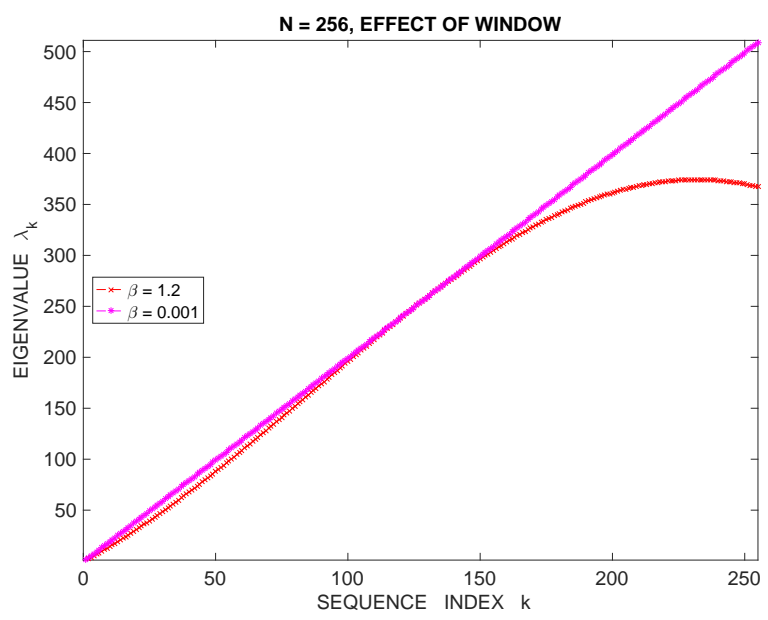

(d)

Figure 2.3: Effect of windowing eigenvalue sequence: (a) DFRFT spectrum magnitude for Kaiser window with $N=256, \beta=15$, (b) DFRFT spectrum magnitude for Kaiser window with $N=$ $256, \beta=0.001$, (c) DFRFT spectral magnitude for $\beta=1.2$, and (d) eigenvalue spectrum with different Kaiser window parameters. Note that we obtain steeper slopes on the peaks of the DFRFT magnitude spectrum for $\beta=1.2$ and for $\beta=15$ we observe a significant amount of aliasing. 


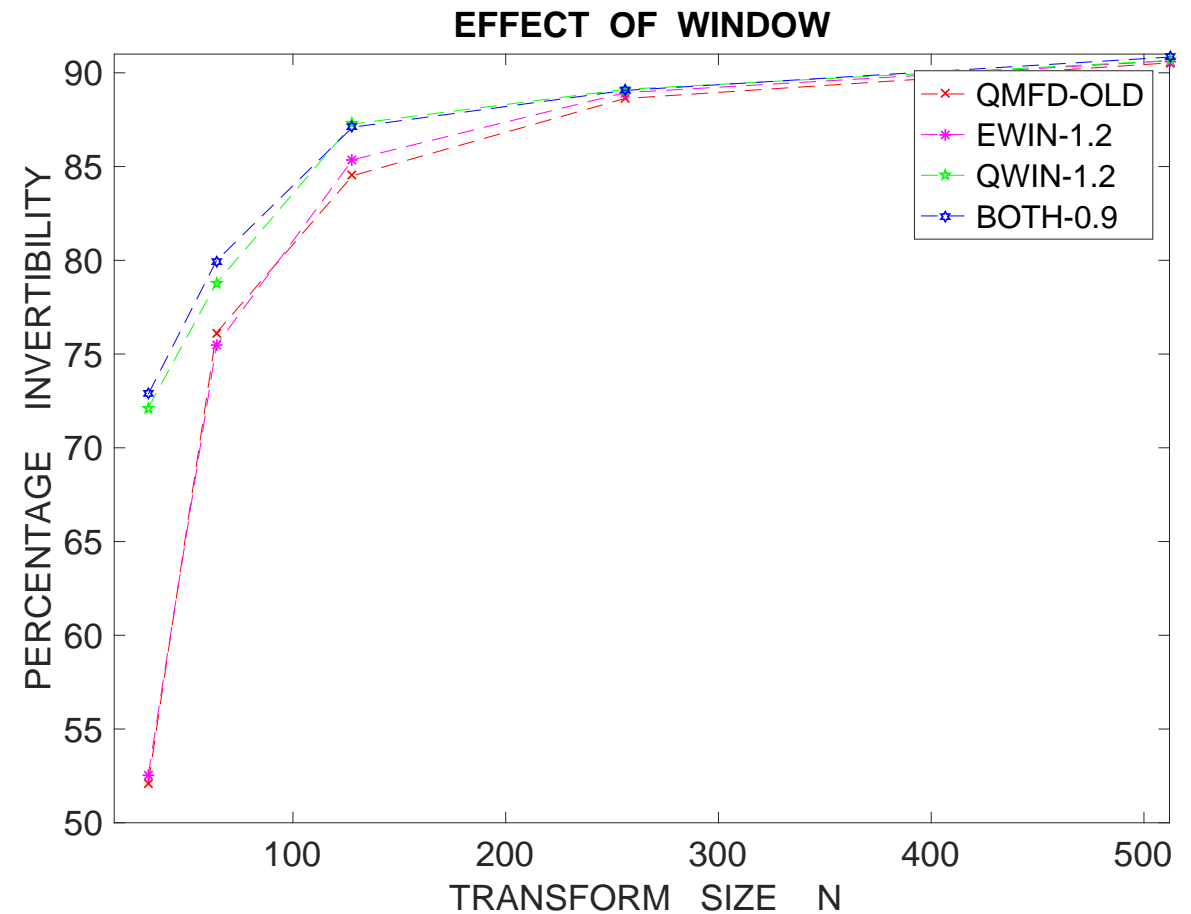

Figure 2.4: Invertibility improvement: Percentage of invertible pixels in the peak to parameter mapping for $\mathbf{Q}$-windowing alone, with a Kaiser window with parameter $\beta=1.2$, for eigenvalue windowing alone, using a Kaiser window with $\beta=1.2$, and combined windowing using a Kaiser window with parameter $\beta_{1,2}=0.9$

mapping compared to other option. Furthermore, we see significant improvement using the windowing approach for smaller transform sizes $N$ because the distortion caused by truncation effects for more pronounced in smaller values for $N$, where the invertibility approaches $91 \%$ for larger matrix sizes $N$.

Invertibility of the mapping as pointed out in [19], impacts the MSE of the corresponding chirp parameter estimates which is shown in Fig. (2.5). The MSE here was calculated at each SNR using 1000 chirps of length $N=32$ in the safe range of $|\alpha|(N-1)+|\omega|=I F<0.85 \pi$.

\subsection{Conclusion}

In this chapter, we have studied the problem of discretizing the Gauss-Hermite operator and the two basic sources of distortion: (a) eigenvalue truncation resulting in spectral distortion of the eigenvalue sequence, and (b) sampling of the eigenfunctions resulting in eigenvector aliasing. We studied two classes of matrices 


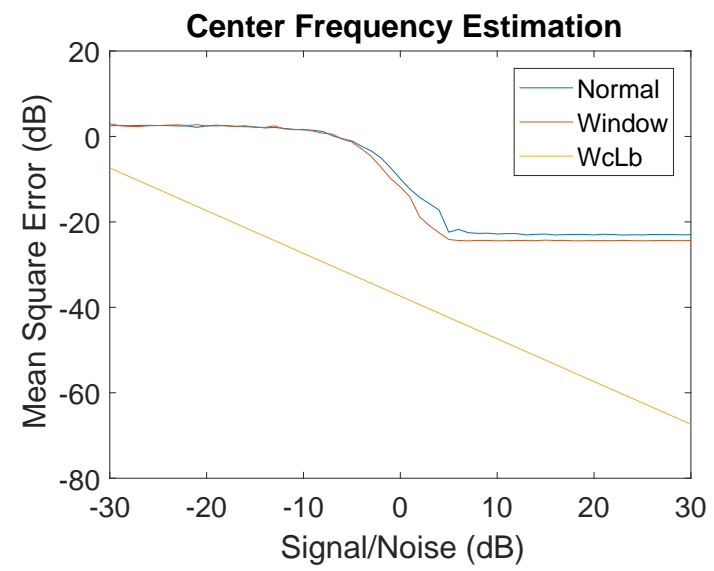

(a)

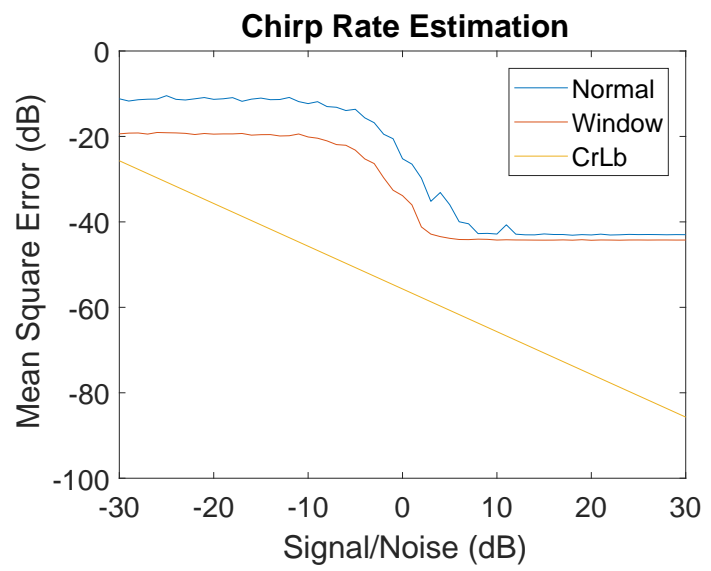

(b)

Figure 2.5: $(\mathrm{a}, \mathrm{b})$ Impact of windowing on the MSE of parameter estimates from the minimumnorm algorithm, depicting improved invertibility of the underlying peak to parameter mapping with combined Q-matrix and eigenvalue windowing.

that commute with DFT/CDFT in the context of the QMFD method developed in [13] in terms of the distortion introduced in discretizing the continuous FRFT.

We studied the quasi-Toeplitz framework where the corresponding $\mathbf{Q}$ matrix is diagonal and developed a weakly non-stationary time-series viewpoint to show the distortion due to discretization. We then studied means of minimizing this distortion by $\mathbf{Q}$ windowing. Using an Kaiser-windowed version of the truncated odd integer valued eigenvalue spectrum, resulted in sharper peaks in the underlying DFRFT spectra in comparison to the boxcar windowed spectra which eventually translated to a wider invertibility region for the underlying peak to parameter mappings. This improvement attained by using windowing is specifically more pronounced for smaller matrix sizes, where the distortion due to discretization is more pronounced.

The main issue with this approach is computational complexity when using a relatively large signal length. The execution time when the signal length $N$ is 512 or higher is not practical for use i.e. it is very time-consuming. So, we looked at developing analytical expressions for the peak to parameter mapping to avoid this complexity that are discussed in the following chapter. 


\section{Chapter 3}

\section{Analytical Expressions for DFRFT Chirp Parameter \\ Estimates}

In this chapter, we look at the application of the MA-CDFRFT to a linear chirp signal. We also look different relations between the peak location and the chirp parameters developed by Vargas-Rubio [20], Peacock [5], and Ishwor [19] and also propose an improved analytical expression for the peak to parameter mapping instead of empirical estimation that was discussed in previous chapter. We further look at the various refinements to this estimator such as zero padding, interpolation, and CZT zooming and express the improvement in terms of MSE over a range of SNR values. Finally, we combine all of the methods for a joint refinement approach to obtain the minimum possible MSE for the center frequency and chirp rate parameters separately, in an attempt to get closer or reside on the CRLB. 


\subsection{Multi-angle Centered FRFT}

Vargas-Rubio and Santhanam [20] discussed the multi-angle CDFRFT via a eigenvalue decomposition for the DFRFT matrix as

$$
\left\{\mathbf{A}_{\alpha}\right\}_{k n}=\sum_{p=0}^{N-1} v_{k p} v_{n p} e^{-\jmath p \alpha}
$$

where $v_{k p}$ is the $\mathrm{k}^{\text {th }}$ element of the $\mathrm{p}^{\text {th }}$ eigenvector. Multiplying $\mathbf{A}_{\alpha}$ by the signal $\mathrm{x}[\mathrm{n}]$, we obtain

$$
\mathbf{X}_{\alpha}[k]=\sum_{n=0}^{N-1} x[n] \sum_{p=0}^{N-1} v_{k p} v_{n p} e^{-\jmath p \alpha} .
$$

For the discrete set of angles $\alpha=\alpha_{r}=\frac{2 \pi r}{N}, r=0,1, \ldots \ldots \ldots, N-1$

$$
\mathbf{X}_{k}[r]=\sum_{p=0}^{N-1} z_{k}[p] \mathbf{W}_{N}^{p r}
$$

where

$$
z_{k}[p]=v_{k p} \sum_{n=0}^{N-1} x[n] v_{n p} .
$$

Expressing the transform as a DFT allows us to use a radix-2 FFT algorithm to compute the CDFRFT. The resulting transform $\mathbf{X}_{k}[r]$ containing the CDFRFT for these discrete angles is called multi-angle DFRFT (MA-CDFRFT).

It is important to observe that $\mathbf{X}_{k}[0]$ corresponds to the original signal $\mathrm{x}[\mathrm{n}]$ with $\mathrm{k}$ $=\mathrm{n}$, and $\mathbf{X}_{k}\left[\frac{N}{4}\right]$ corresponds to the CDFT of $\mathrm{x}[\mathrm{n}]$ when $\mathrm{N}$ is a multiple four, since in this case, $\alpha_{\frac{N}{4}}=\frac{\pi}{2}$. Fig. (3.1) shows a graphical representation of the array $\mathbf{X}_{k}[r]$ to illustrate how the interpretation of index $k$ is dependent on the value of $r$. For example, when $r=0, k$ is interpreted as time and when $r=\frac{N}{4}, k$ is interpreted as frequency instead. This interpretation also shows that the upper half of $\mathbf{X}_{k}[r]$ is nothing but a reversed version of the lower half. So, just by calculating the lower half, the upper half is known as well. 


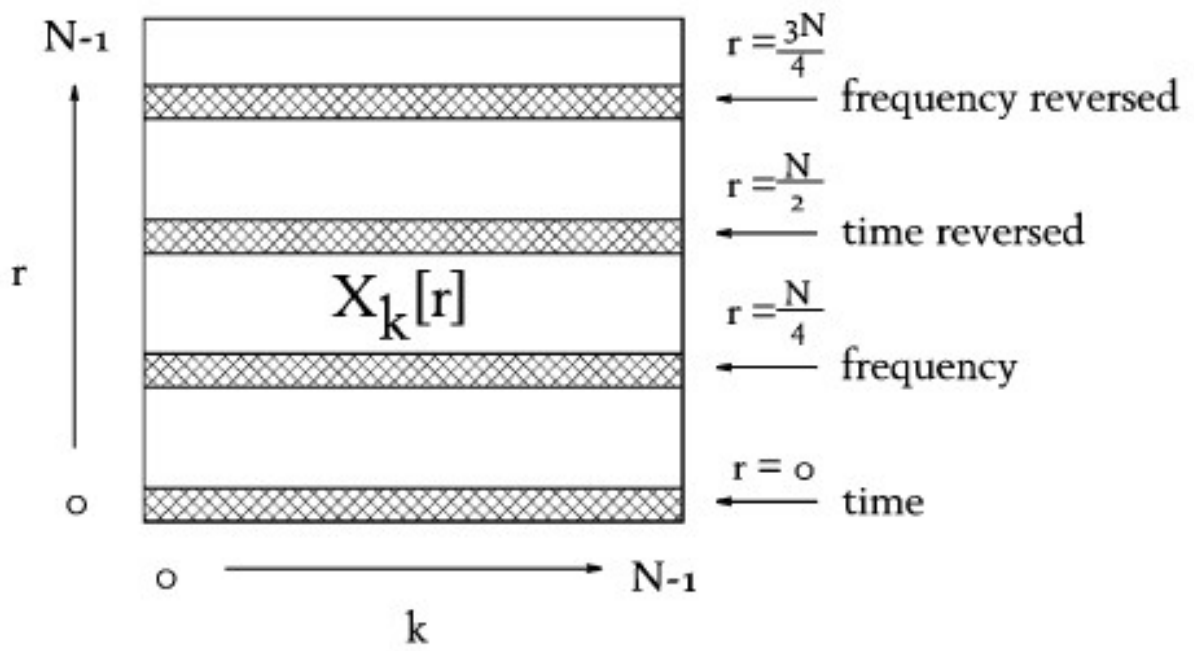

Figure 3.1: Graphical representation of $\mathbf{X}_{k}[r]$ that shows how the interpretation of the index $\mathrm{k}$ changes depending on the value of index $r$ [20].

\subsection{Linear Chirp Signal}

The kernel of the FRFT containing a linear chirp signal is one of the most relevant properties of the FRFT. So,the MA-CDFRFT can be applied for the estimation of the chirp parameters of a single-component (complex) signal or multi-component (real) signal. Usually, a complex chirp signal is of the form

$$
x[n]=A e^{j\left(\omega_{o} n+c_{r} m^{2}\right)}, \quad 0 \leq n \leq N-1 \text { and } m=n-\frac{N-1}{2}
$$

where $c_{r}$ is the chirp rate and $\omega_{o}$ is the center frequency and $A$ is the amplitude of the signal. Upon application of the MA-CDFRFT to this signal, we obtain a sharp peak in the chirp rate versus frequency representation as shown in Fig. (3.2). For the purpose of this thesis, we used $c_{r}=0.001, \omega_{o}=\pi / 3$ and $N=128$. We see the peak location at the coordinates $(86,34)$. At this coordinate, the magnitude of the MA-CDFRFT is a maximum. Specifically, we can observe that we actually have two maxima, because the CDFRFT at $\alpha+\pi$ is the reversed version of CDFRFT at $\alpha$. The location of the other maxima is $(42,98)$.

Similarly, for a multi-component chirp signal of the form

$$
x[n]=A \cos \left(\omega_{o} n+c_{r} m^{2}\right)=A \frac{1}{2}\left(e^{j\left(\omega_{o} n+c_{r} m^{2}\right)}+e^{-j\left(\omega_{o} n+c_{r} m^{2}\right)}\right),
$$




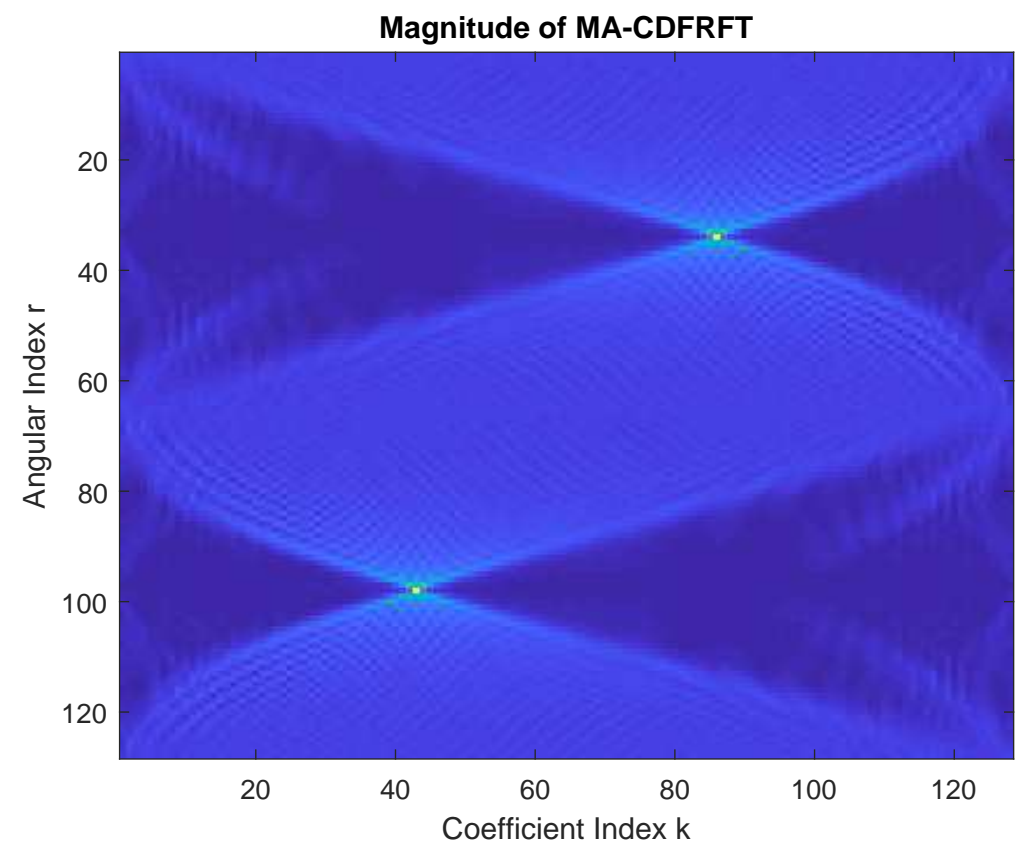

Figure 3.2: Magnitude of the MA-CDFRFT for $c_{r}=0.001, \omega_{o}=\pi / 3$ and $N=128$ and QMFD basis for complex chirp signal.

where the symbols mean and have same value as in Eq. (3.5). This signal consists of two linear chirps, one with a positive chirp rate, and another with a negative chirp rate. Both signal have same center frequency. As shown in Fig. (3.3), the magnitude of the transform displays four peaks, two for each signal, and thus it can be clearly deduced that the signal consists of two linear chirp components. For our further experiments and for the sake of uniformity, we will use this real chirp signal and consider the location of the top-left peak all the time.

\subsection{Relation between Peak and Parameter}

Once the peak locations are obtained, we can apply the peak-to-parameter mapping to get the parameter estimates. Rubio-Santhanam [20] derived an empirical relation between the chirp parameters and the angle $\alpha$ as

$$
\begin{aligned}
& c_{r} \approx 2 \frac{\tan (\alpha-\pi / 2)}{N}+1.41 \frac{(\alpha-\pi / 2)}{N} \\
& \omega_{0}=\omega_{p}+0.85(\alpha-\pi / 2)^{3},
\end{aligned}
$$




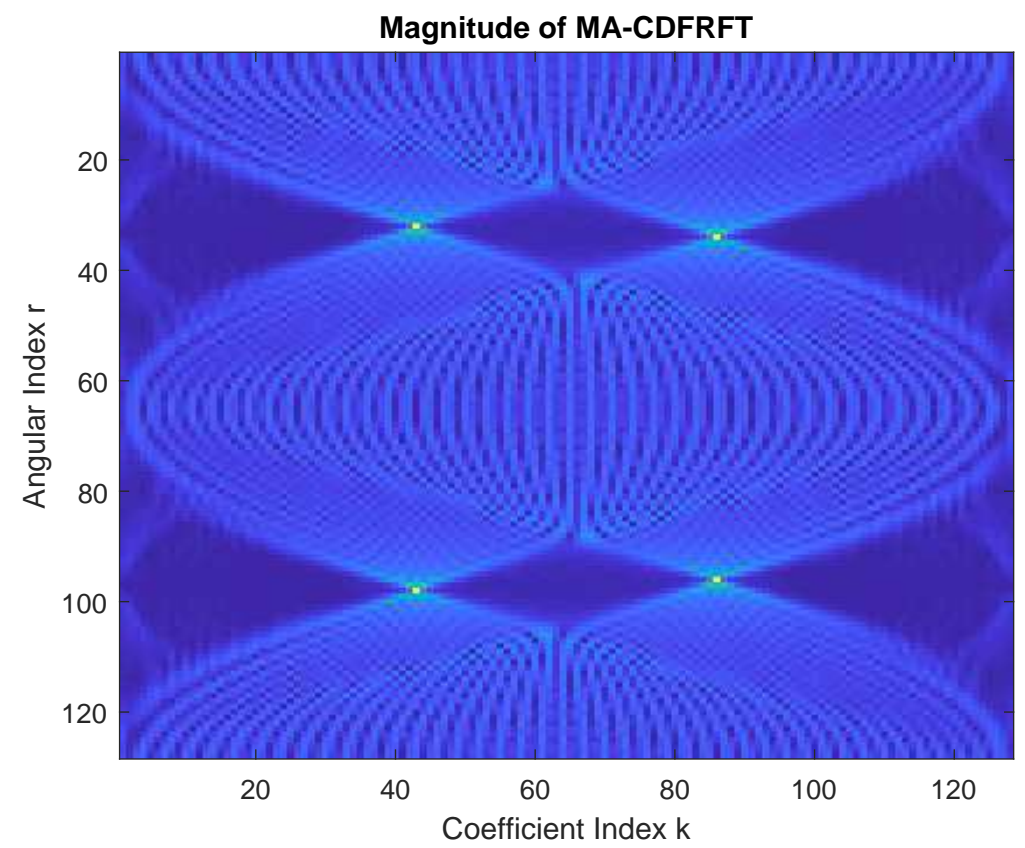

Figure 3.3: Magnitude of the MA-CDFRFT for $c_{r}=0.001, \omega_{o}=\pi / 3$ and $N=128$ and QMFD basis for real chirp signal.

where $\omega_{0}$ is the average frequency, and $\omega_{p}$ is the frequency at which the peak of the CDFRFT occurs. This method was used to estimate the parameters if the angle of the transform at which peak occurred was known.

Peacock and Santhanam [5] derived an expression for the mapping as

$$
\begin{aligned}
& \alpha=-\frac{\pi}{N} \cot \left(\frac{k \pi}{N}\right) \\
& \omega=\frac{2 \pi}{N}\left(r-\frac{N-1}{2}\right) \csc \left(\frac{k \pi}{N}\right) .
\end{aligned}
$$

But Ishwor and Santhanam [19] found out that this mapping does not correctly map the peak locations to the corresponding chirp rate and center frequency. They modified this peak to parameter mapping expression to correctly map the peak location as

$$
\begin{aligned}
& \hat{c}_{r}=-\frac{\pi}{N} \cot \left(\frac{2 r_{p} \pi}{N}\right) \\
& \hat{\omega}_{c}=\frac{2 \pi}{N}\left(k_{p}-\frac{N-1}{2}\right) \csc \left(\frac{2 r_{p} \pi}{N}\right),
\end{aligned}
$$

where $\hat{c}_{r}$ is the chirp rate, $\hat{\omega}_{c}$ is the center frequency and $\left(k_{p}, r_{p}\right)$ is the peak location. They concluded that using this peak-to-parameter mapping results in 
error of about $0.4 \%$ for the QMOD method [19].

However, this estimator does not consider that fact that for an even $N$, the basis vectors of the CDFT have a frequency that falls exactly in the middle of the DFT. This means that when computing the CDFT, there is no coefficient for zero frequency (average value of the signal), and in some cases this may be an issue. This problem can be solved by shifting the signal to be transformed by a frequency of $\pm \pi / N$ [20]. This is achieved by multiplying the signal by a complex signal $e^{j \pi n / N}$. We modified the estimator to incorporate this frequency shift as

$$
\begin{aligned}
& \hat{c}_{r}=-\frac{\pi}{N} \cot \left(\frac{2\left(r_{p}-\frac{1}{2}\right) \pi}{N}\right) \\
& \hat{\omega}_{c}=\frac{2 \pi}{N}\left(k_{p}-\frac{N-1}{2}-\frac{1}{2}\right) \csc \left(\frac{2\left(r_{p}-\frac{1}{2}\right) \pi}{N}\right),
\end{aligned}
$$

which results in

$$
\begin{aligned}
& \hat{c}_{r}=-\frac{\pi}{N} \cot \left(\frac{2 \pi}{N}\left(r_{p}-\frac{1}{2}\right)\right) \\
& \hat{\omega}_{c}=\frac{2 \pi}{N}\left(k_{p}-\frac{N}{2}\right) \csc \left(\frac{2 \pi}{N}\left(r_{p}-\frac{1}{2}\right)\right) .
\end{aligned}
$$

In other words, we can say that this "half-sample" shift in these expressions is to account for the shift in the origin of the CDFT frequency measurements when $N$ is an even integer.

\subsection{MA-CDFRFT: Zero padding, Spectral Peak Interpolation and CZT Zoom}

\subsubsection{Parameter Estimation via the CDFT and Zero padding}

Zero padding is a technique used in spectral analysis for interpolating peaks. It basically extends the signal or spectrum with zeros. It maps the length of signal $\mathrm{N}$ to $\mathrm{M}$ (where $\mathrm{M}$ is larger than $\mathrm{N}$ ) adding equal amount of zeros at both ends of the signal. 


\section{Definition:}

$$
x_{z e r o p a d}(M)=[z \operatorname{eros}(M / 2) \quad x(N) \quad z \operatorname{eros}(M / 2)] .
$$

For example, if $x=[1,2,3,4,5,6]$ then

$$
x_{\text {zeropad }}(10)=[0,0,1,2,3,4,5,6,0,0] .
$$

Zero padding allows us to use a longer CDFT, which in turn produces a longer CDFT result vector. A longer CDFT result has more frequency bins that are more closely spaced in frequency. This results in a smoother looking spectrum when plotted with further zero padding. Although this will not help with resolving or the resolution of and/or between adjacent nearby frequencies, it makes it easier to visually resolve a single peak at a single isolated frequency that does not have any significant adjacent signals or noise in the spectrum. The analytical expressions change when incorporating the zero padding and become:

$$
\begin{aligned}
\hat{c}_{r} & =-\frac{\pi}{M} \cot \left(\frac{2 \pi}{M}\left(r_{p}-\frac{1}{2}\right)\right) \\
\hat{\omega}_{c} & =\frac{2 \pi}{M}\left(k_{p}-\frac{M}{2}\right) \csc \left(\frac{2 \pi}{M}\left(r_{p}-\frac{1}{2}\right)\right),
\end{aligned}
$$

where $M$ is the zero padded signal length. The signal into consideration is a real multi-component signal is Eq. (3.6) where $A$ is the amplitude, $\omega_{c}$ is the center frequency, $c_{r}$ is the chirp rate and $w[n]$ is AWGN of standard deviation $\sigma$. For the purpose of this thesis, we set the parameters as

$$
N=128, \quad A=1, \quad \omega_{c}=\pi / 3, \quad \text { and } \quad c_{r}=0.001
$$

In order to show how zero padding works, we present a numerical example here. Here, this observation with noise has 128 samples. Applying the MA-CDFRFT algorithm, the peak location is at $(32,43)$ coordinate. From Eq. (3.11), we get $\hat{\omega}_{c}=1.0311$ and $\hat{c}_{r}=0.00060251$. If we use a $M=256$ point CDFT to estimate the parameters, the ccordinated of the peak is located at $(62,86)$ and the estimates are $\hat{\omega}_{c}=1.03277$ and $\hat{c}_{r}=0.0007537$. Further zero padding, i.e. if we use a 


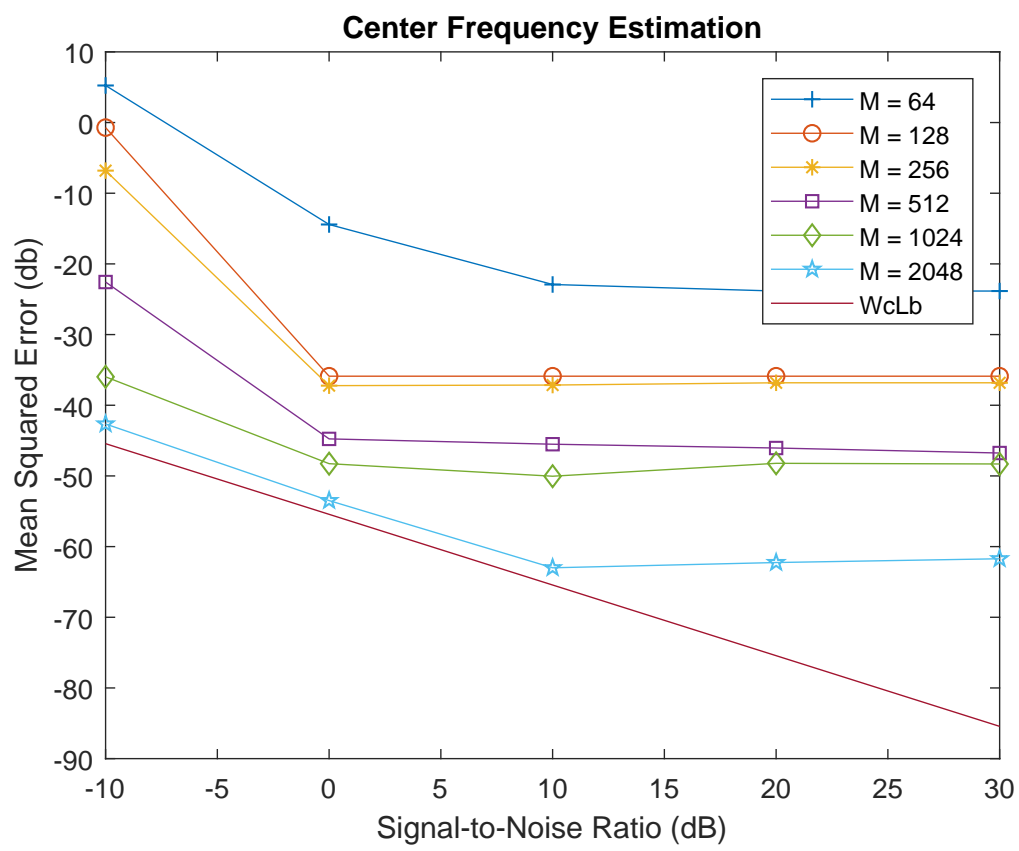

(a)

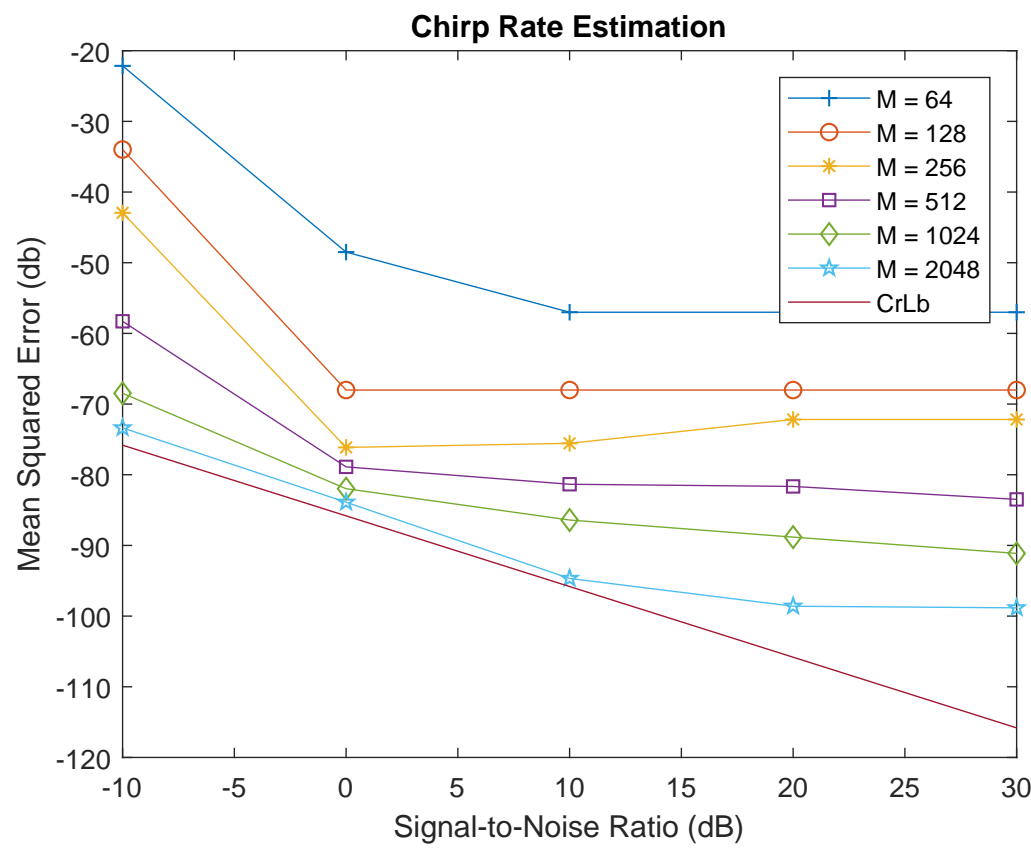

(b)

Figure 3.4: Performance of the chirp parameter estimators based on the analytical expression and zero padding in DFRFT in comparison to the CRLB, obtained by averaging over 200 experiments. The basis of CDFT eigenvectors used in the computation of the MA-CDFRFT spectrum was the QMFD basis. 
$M=512$ point CDFT, the peak is located at $(116,172)$ thus yielding the estimates $\hat{\omega}_{c}=1.0425$ and $\hat{c}_{r}=0.000925$. There is an improvement in the MSE associated with both of the analytical expressions with respect to the CRLB as the level of zero-padding increases or size of CDFT increases. This is a direct consequence of better peak resolution resulting from spectral interpolation.

Fig. (3.4) depicts the MSE of the center frequency and chirp rate estimates respectively of the zero padding approach as a function of SNR for multiple values of $M, M=64, M=128, M=256, M=512, M=2014$, and $M=2048$. If we observe the plot, we can see that there is improvement of about $12 \mathrm{~dB}$ for every quadruple increase in the length of $M$. So, theoretically, if we kept on increasing the size of CDFT or zero padding over $M=2048$, the MSE will hit the CRLB and we can attain the CRLB asymptotically. Finally, according to [33], we can say that, this estimator is similar to MLE.

\subsubsection{Parameter Estimation via the CDFT and Spectral Peak Interpolation}

This is a two stage method to calculate the parameter estimates using three CDFT samples at the peak. The first stage is to calculated the peak location using 2D peak estimator. The second stage is to perform a fine search around the peak obtained from the first stage. This method suggests a nonlinear relation involving three samples to a real valued fine resolution of the peak, and thus better chirp parameter estimates.

In the first stage, we calculate the peak locations $(r, k)$. Then, the N-point DFT of the signal is calculated at these points, $A_{k}=\sum_{n=0}^{N-1} x[n] W_{N}^{n k}$ and $A_{r}=$ $\sum_{n=0}^{N-1} x[n] W_{N}^{n r}$. Here $A_{k}$ and $A_{r}$ denotes the complex valued DFT output. The peak value in the DFT magnitude spectrum $\left(r_{p}, k_{p}\right)$ is expected to be around the true parameters $\omega_{p}$ and $c r_{p}$. Then, we can express the DFT bins where the peak 
occurs and its immediate left and right neighbors as:

$$
\begin{aligned}
A_{k-1} & =\sum_{n=0}^{N-1} x[n] W_{N}^{n(k-1)}, \\
A_{k} & =\sum_{n=0}^{N-1} x[n] W_{N}^{n k}, \\
A_{k+1} & =\sum_{n=0}^{N-1} x[n] W_{N}^{n(k+1)} .
\end{aligned}
$$

Similarly, for $r$ axis, we have:

$$
\begin{aligned}
A_{r-1} & =\sum_{n=0}^{N-1} x[n] W_{N}^{n(r-1)}, \\
A_{r} & =\sum_{n=0}^{N-1} x[n] W_{N}^{n r}, \\
A_{r+1} & =\sum_{n=0}^{N-1} x[n] W_{N}^{n(r+1)} .
\end{aligned}
$$

Now, we use three different interpolation techniques for fine resolution peak location estimation.

- Parabolic Interpolation [21]:

$$
\begin{aligned}
& \hat{\delta}_{k}=\left(\left|A_{k+1}\right|-\left|A_{k-1}\right|\right) /\left(4\left|A_{k}\right|-2\left|A_{k-1}\right|-2\left|A_{k+1}\right|\right), \\
& \hat{\delta}_{r}=\left(\left|A_{r+1}\right|-\left|A_{r-1}\right|\right) /\left(4\left|A_{r}\right|-2\left|A_{r-1}\right|-2\left|A_{r+1}\right|\right) .
\end{aligned}
$$

- Candan Interpolation [22]:

$$
\begin{gathered}
\hat{\delta}_{k}=\frac{\tan (\pi / N)}{\pi / N} \operatorname{Real}\left\{\left(A_{k-1}-A_{k+1}\right) /\left(2 A_{k}-A_{k-1}-A_{k+1}\right)\right\}, \\
\hat{\delta}_{r}=\frac{\tan (\pi / N)}{\pi / N} \operatorname{Real}\left\{\left(A_{r-1}-A_{r+1}\right) /\left(2 A_{r}-A_{r-1}-A_{r+1}\right)\right\} .
\end{gathered}
$$




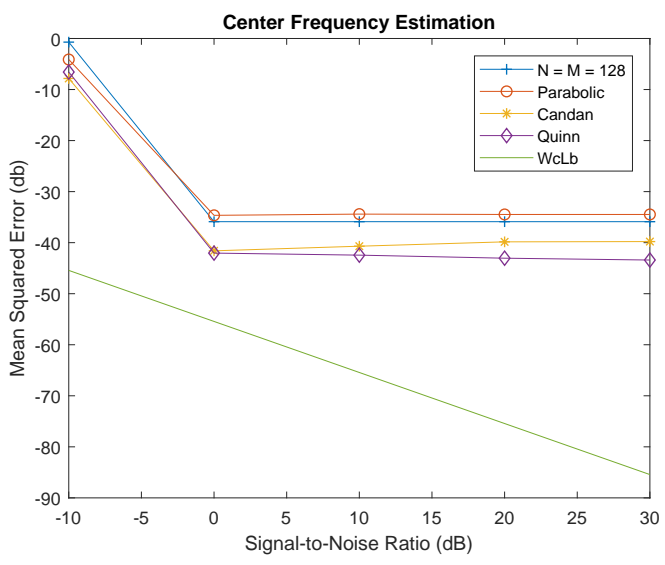

(a)

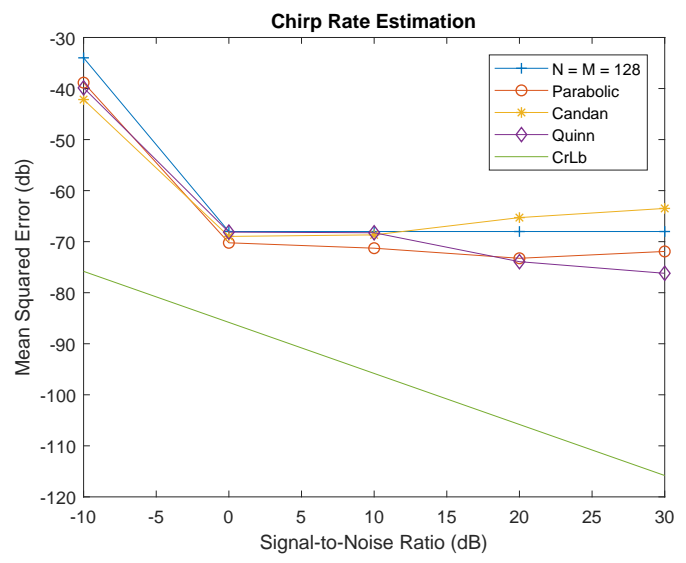

(b)

Figure 3.5: Effects of interpolation on the chirp parameter estimates (a,b) comparison of various forms of peak interpolation with $\mathrm{N}=\mathrm{M}=128$.

- Quinn Interpolation[23]:

$$
\begin{aligned}
& \alpha_{1}=\operatorname{Real}\left(A_{k-1} / A_{k}\right), \alpha_{2}=\operatorname{Real}\left(A_{k+1} / A_{k}\right), \\
& \delta_{1}=\alpha_{1} /\left(1-\alpha_{1}\right), \delta_{2}=\alpha_{2} /\left(1-\alpha_{2}\right), \\
& \text { if } \delta_{1}>0 \text { and } \delta_{2}>0, \hat{\delta}_{k}=\delta_{2} \text { else } \hat{\delta}_{k}=\delta_{1} . \\
& \text { And } \\
& \beta_{1}=\operatorname{Real}\left(A_{r-1} / A_{r}\right), \beta_{2}=\operatorname{Real}\left(A_{r+1} / A_{r}\right), \\
& \delta_{1}=\beta_{1} /\left(1-\beta_{1}\right), \delta_{2}=\beta_{2} /\left(1-\beta_{2}\right), \\
& \text { if } \delta_{1}>0 \text { and } \delta_{2}>0, \hat{\delta}_{r}=\delta_{2} \text { else } \hat{\delta}_{r}=\delta_{1} .
\end{aligned}
$$

The peak coordinates are then modified as:

$$
k_{p}=k+\hat{\delta}_{k} \quad \text { and } \quad r_{p}=r+\hat{\delta}_{r}
$$

Fig. (3.5) depicts the MSE of both parameters as a result of applying spectral peak interpolation approaches on the peak of the signal. In the first case $(a, b)$ signal of length $N=128$ is used. Quinn's Interpolation produces the best results for center frequency estimation however for the chirp rate, the improvements are minimal. 


\subsubsection{Parameter Estimation via the CDFT and CZT Zoom}

The CZT-based zoom operation produces zooming in the angle or chirp rate variable $r$ and only affects the chirp rate estimator and not the center frequency estimator. There are two ways to apply CZT zoom on the MA-CDFRFT algorithm. First, the $(0,2 \pi)$ range of the $r$-axis is zoomed in to $\left(0, \alpha * \frac{\pi}{2}\right)$ where $\alpha=1,2,3, \ldots$ and second, the range is zoomed into the interval (upperlim, upperlim $+\pi / 2$ ). Here, upperlim is dependent on the zooming factor by,

$$
\text { upperlim }=\pi / 2-\pi / \text { zoom }
$$

In the first case, CZT zoom limits the search in the $r$-axis, thus decreasing the size of the axis itself which in turn increases the $r$-index number. In order to incorporate this change, some modification has to be made in the estimators and they are:

$$
\begin{aligned}
& \hat{c}_{r}=-\frac{\pi}{M} \cot \left(\frac{2 \pi}{M * z o o m}\left(r_{p}-\frac{1}{2}\right)\right) \text { and } \\
& \hat{\omega}_{c}=\frac{2 \pi}{M}\left(k_{p}-\frac{M}{2}\right) \csc \left(\frac{2 \pi}{M * \text { zoom }}\left(r_{p}-\frac{1}{2}\right)\right),
\end{aligned}
$$

where zoom is the factor by which we zoom into the $r$-axis. While in the second case, the starting point of the $r$-axis is no longer zero, we have to scale back the peak locations to the $(0,2 \pi)$ range and use the analytical expression to calculate the parameters. If the peak locations after tge CZT zoom is $(r, k)$ the Eq. (3.10) can be used after scaling back the locations by,

$$
r_{p}=1+\frac{\text { upperlim }+(r-1) * \frac{2 \pi}{M * z o o m}}{2 \pi / M} \text { and } \quad k_{p}=k
$$

In this thesis, we employ the first CZT zoom method. We cannot go beyond 4-times zoom using this method, however, the second method allows us to zoom beyond that as well. Fig. (3.6) shows the results of applying CZT-based zoom on the MSE associated with the chirp parameters. We can see that the improvement is primarily associated with the chirp rate but not the center frequency. There is 


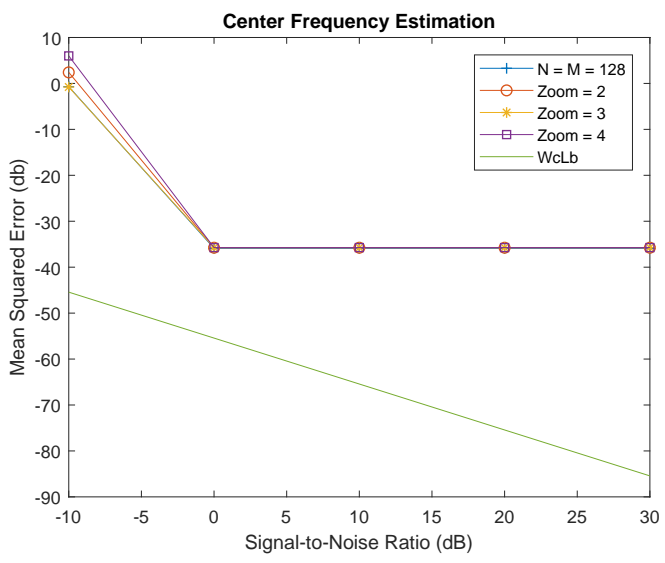

(a)

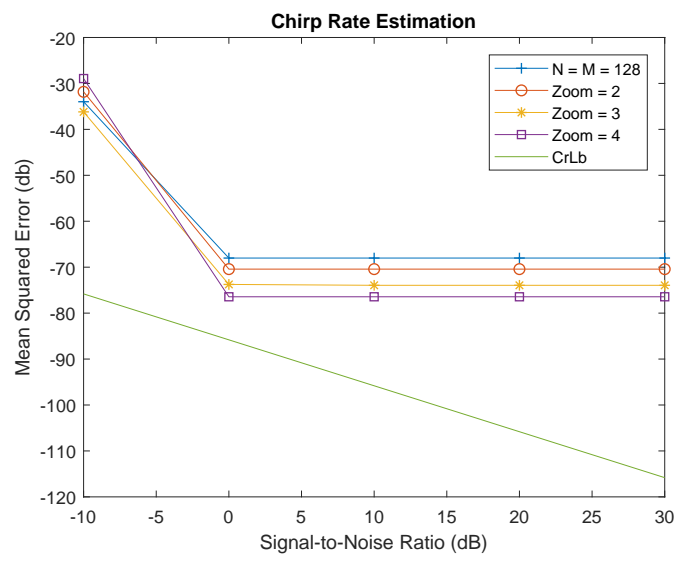

(b)

Figure 3.6: $(\mathrm{a}, \mathrm{b})$ Effect of CZT-based zoom on the chirp parameter estimates for $\mathrm{N}=\mathrm{M}=128$

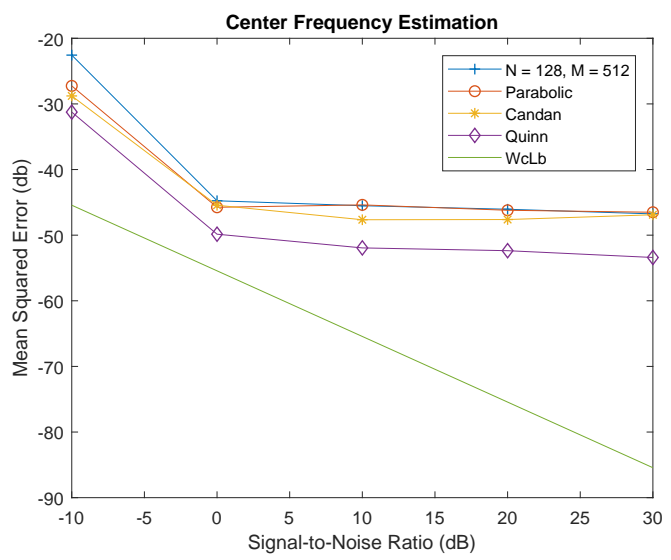

(a)

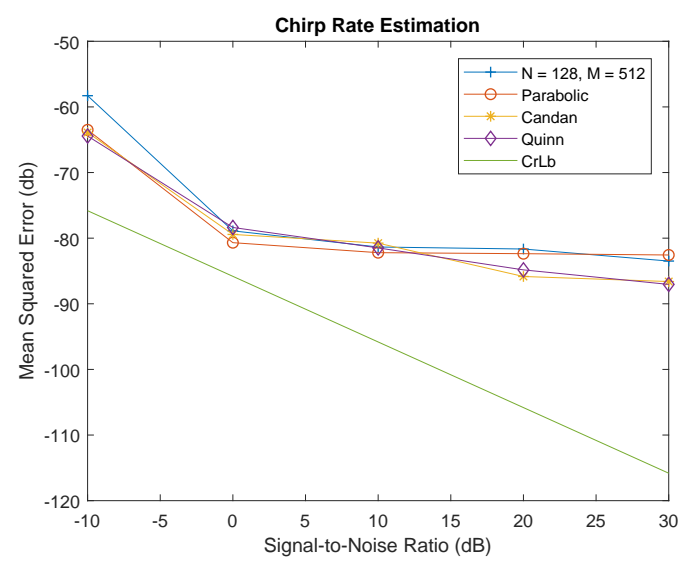

(b)

Figure 3.7: (a,b) Effect of zero padding and interpolation combined for $\mathrm{N}=128$ and $\mathrm{M}=512$ followed by interpolation

a consistent improvement obtained by increasing the zooming factor in the MSE of the chirp rate estimates.

\subsubsection{Combination of the Refinements}

We saw improvement in the MSE plot for all three refinement approaches. Ideally, combining all the three techniques to form a joint refinement technique would result in better estimate compared to applying single one of the approach. So, we experiment with different combinations of these refinement techniques.

First, we look at the combination of zero padding and the spectral peak interpolation technique. As seen in previous case, a chirp length of $N=128$ and CDFT size 


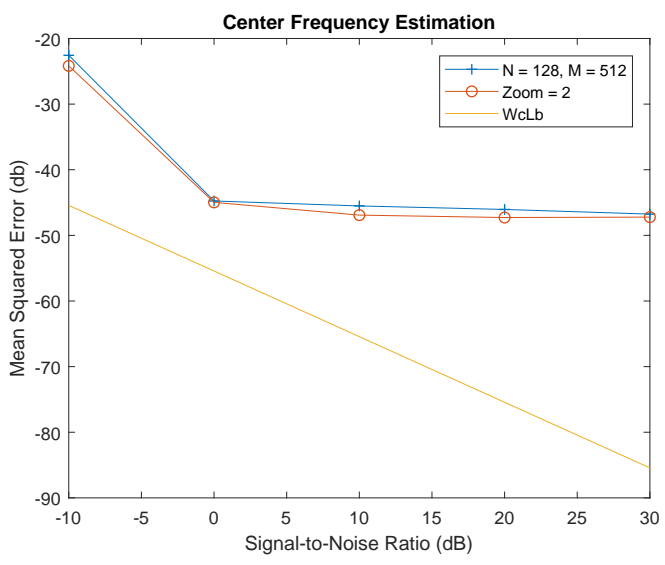

(a)

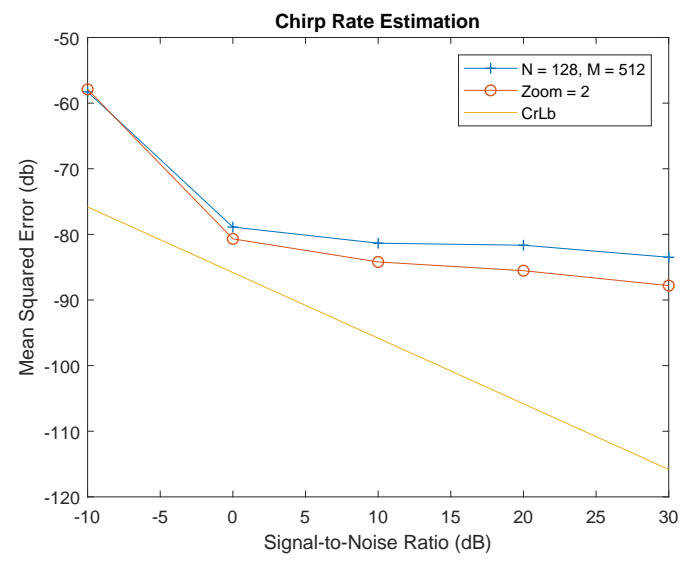

(b)

Figure 3.8: (a,b) Effect of zero padding and CZT zoom for $\mathrm{N}=128$ and $\mathrm{M}=512$ followed by CZT zoom

of $M=512$ produced significant improvement in the MSE so did spectral peak interpolation technique. Fig. (3.7) shows the MSE of the estimated chirp parameters upon applying this combination. The improvement in the MSE for center frequency is more pronounced compared to the chirp rate for Quinn's interpolation technique combined with zero padding, as for chirp rate, the improvement is minimal.

Next, we try various combinations of zero padding and CZT zoom. Again, the chirp length or signal length is maintained $N=128$ and CDFT size of $M=512$ and a zooming factor of 2 is taken into consideration. Fig. (3.8) shows the MSE plots for the chirp parameters after applying this combination. As expected, the improvement is prominent in the chirp rate parameter as the CZT zoom occurs in $r$-axis. The center frequency estimate shows a slight improvement because of the better $r$-coordinate obtained after CZT zoom.

The last step is to combine all three techniques, i.e. zero padding followed by CZT zoom and then spectral peak interpolation on the CZT zoomed peak coordinates. The $N, M$ and zoom factor are kept the same as above. We have used Quinn's interpolation since it resulted in the best performance. Fig. (3.9) shows the MSE plots for this combination. We can see that there is a vast improvement in terms of MSE for center frequency parameter using the combination of all three methods. However, the same is not true for chirp rate, which has the best MSE results for 


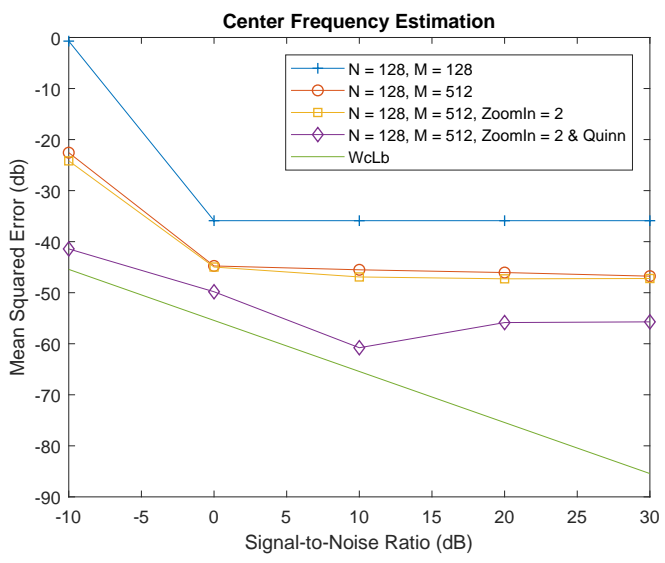

(a)

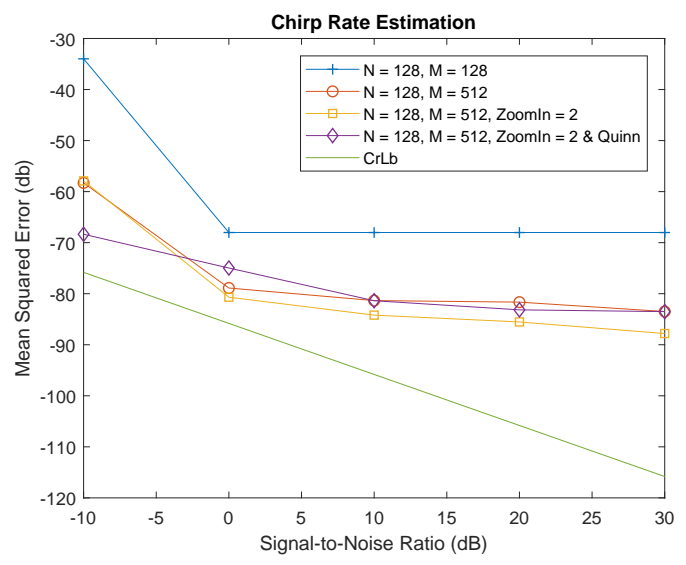

(b)

Figure 3.9: $(\mathrm{a}, \mathrm{b})$ Effect of zero padding for $\mathrm{N}=128$ and $\mathrm{M}=512$ followed by CZT zoom and Quinn's spectral peak interpolation

combination of zero padding and CZT zoom.

So, we conclude that the combination of zero padding, Quinn's spectral peak interpolation technique, and CZT zoom results in the best possible estimate for the center frequency. The reason is that this combination yields the lowest MSE when compared with the CRLB. However, for the purposes of chirp rate estimation, the combination of zero padding and CZT zoom gives the lowest MSE.

To sum up the discussion, Fig. (3.10) shows the MSEs of the chirp parameter estimates using the plain vanilla analytical expressions and the analytical expressions combined with all the refinements. We can see the vast improvement in terms of MSE over a range of SNR. These results are obtained by averaging the MSE over 200 experiments.

\subsection{Conclusion}

In this chapter, we studied the basics of the MA-CDFRFT and its relation with the linear chirp signal. We saw the results of using a single component chirp signal as well as multi-component chirp signal as the input to the MA-CDFRFT algorithm to obtain spectral peaks in the magnitude spectrum. We then studied the relation between these spectral peak and the chirp parameters i.e. the peak- 


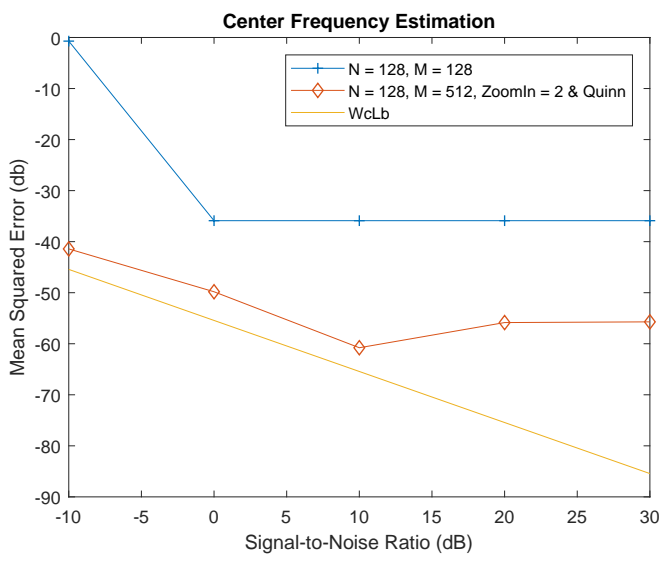

(a)

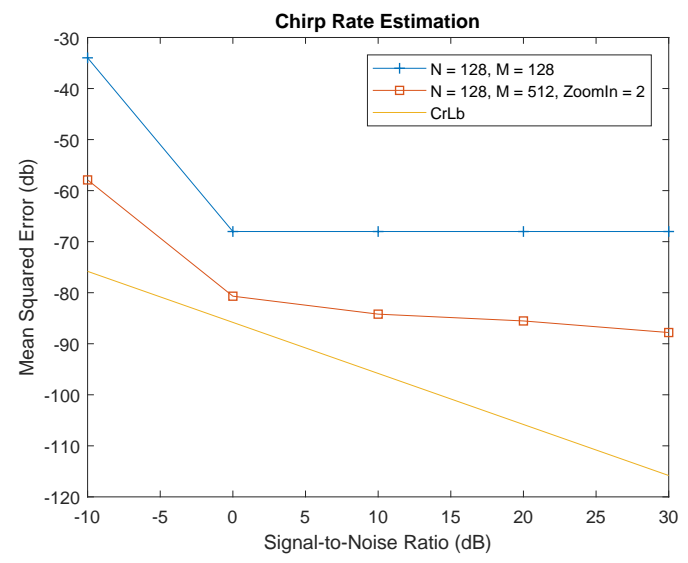

(b)

Figure 3.10: Comparision between the vanilla analytical expression and the final analytical expression with the refinements (a) MSE vs SNR for center frequency (b) MSE vs SNR for chirp rate

to-parameter mapping. Previous methods based this mapping did not consider the problems with even $N$. So, the analytical expressions were modified with a "half-sample" shift to incorporate the coefficient for zero frequency i.e. average value of the signal.

We then investigated different refinements to these expressions such as zero padding, spectral peak interpolation, and CZT zoom. We then combined these refinements and proposed the best combination for the center frequency and chirp rate estimates separately. 


\section{Chapter 4}

\section{Application to Real SAR Data}

\subsection{Introduction}

SAR is a ubiquitous remote-imaging technique utilized in many disciplines [24] [25] [4]. It can be used in all-weather environments since SARs are active sensors which provide their own illumination, and they work in the microwave spectrum where the attenuation of electromagnetic waves is low.

It is a form of radar that is used to create two- or three-dimensional images of objects, such as landscapes. SAR uses the motion of the radar antenna over a target region to provide finer spatial resolution than conventional beam-scanning radars. SAR is typically mounted on a moving platform, such as an aircraft or spacecraft, and has its origins in an advanced form of side looking airborne radar (SLAR). The distance the SAR device travels over a target in the time taken for the radar pulses to return to the antenna creates the large synthetic antenna aperture (the size of the antenna). Typically, the larger the aperture, the higher the image resolution will be, regardless of whether the aperture is physical (a large antenna) or synthetic (a moving antenna) - this allows SAR to create high-resolution images with comparatively small physical antennas.

To create a SAR image, successive pulses of radio waves are transmitted to "illuminate" a target scene, and the echo of each pulse is received and recorded. 
The pulses are transmitted and the echoes received using a single beam-forming antenna, with wavelengths of a meter down to several millimeters. As the SAR device on board the aircraft or spacecraft moves, the antenna location relative to the target changes with time. Signal processing of the successive recorded radar echoes allows the combining of the recordings from these multiple antenna positions. This process forms the synthetic antenna aperture and allows for the creation of higher-resolution images than would otherwise be possible with a given physical antenna [26].

An underlying assumption when forming a SAR image is that all targets on the ground scene are static. Any vibration movement of targets in the range direction in the ground scene engenders a Doppler shift in the returned SAR signal [27]. This shift causes the vibrating target to manifest as a smearing effect in the SAR images. This smearing manifests itself as a ghost image surrounding the vibrating target at the same range line and results in obscuring the actual target shape [4][28]. This ghost image creates vibration signatures that help in target identification, retrieve machinery status, and thus detect prohibited activities. In general, these vibration signatures include transient signals like chirped sinusoids, and their associated base frequencies and chirp rate.

Normally, the vibration signatures of the sources are altered by its housing structure which produces difference between the vibration signature of the source and the vibration signature measured from the exterior. When the concealed vibrating source is a single frequency component sinusoid, in most cases, the reflected signature will be attenuated and delayed with respect to the characteristic of the housing structure. This brings into focus the SNR reduction problem which depends on the frequency of the vibration.

Applying the DFRFT-based technique, we can estimate the acceleration and center frequency components of the vibrating object [4] [28] [30]. This method is reliable when both SNR and SCR are above $15 \mathrm{~dB}$ [29]. But by combining the DFRFT technique with subspace filtering [29] and rank reduction techniques [30], it is possible to relax the requirements to $8 \mathrm{~dB}$ and $5 \mathrm{~dB}$ respectively. 


\subsection{SAR Signal Model}

We assume that while forming the SAR images, all the scatters in the ground scene are static. When this assumption is not satisfied i.e. the target vibrates in the range direction, the SAR returned signals present a smearing effect along the azimuth direction. This is primarily due to a vibration-induced time-varying phase and this can be modeled by using a second-order Taylor expansion of the SAR phase-history signal in short-time windows. From [4], this approximation can be stated as

$$
x[n] \approx \sigma \exp \left[j\left(\phi-\frac{4 \pi f_{c}}{c} r_{d}[m]+\left(f_{y} \bar{y}-\frac{4 \pi f_{c} v_{d}[m]}{c f_{p r f}}\right) n-\frac{2 \pi f_{c} a_{d}[m]}{c f_{p r f}^{2}} n^{2}\right)\right]+w[n],
$$

where $m \leq n \leq m+N_{w}, f_{c}$ is the RF carrier frequency, $c$ is the speed of light, $f_{p r f}$ is the pulse repetition frequency of the SAR system, $w[n]$ is AWGN, $\sigma$ is the target reflectivity (constant), $r_{d}[m], v_{d}[m]$ and $a_{d}[m]$ are the instantaneous position, speed and acceleration respectively of the target in the range direction, $N_{w}$ is the sub-aperture size, $\bar{y}$ is the average azimuth position of the target, $f_{y}$ is an imaging factor given by Eq. (4.2), $n$ is the azimuth bin location, and $m$ is the sub-aperture index. We can see in Eq. (4.1), in a short-time window $x[n]$ is approximately a chirp signal whose chirp rate is linearly proportional to the instantaneous vibration acceleration $a_{d}[m]$

$$
f_{y}=\frac{4 \pi f_{c}}{c} \frac{V}{R_{0} f_{p r f}}
$$

where $V$ is the nominal speed of the SAR antenna, and $R_{0}$ is the distance from the patch center to the mid-aperture.

\subsection{Vibration Estimation}

Using the DFRFT technique and the signal model of Eq. (4.1), we can estimate the instantaneous acceleration of the vibrating object. In this method, the signal 
is first approximated by a chirp signal by applying the DFRFT in successive overlapping time windows called sub-apertures. In each of these sub-apertures, we are using the analytical expression to calculate the chirp rate from the peak locations. The acceleration of the vibrating target is dependent on the chirp rates estimated at each sub-apertures as

$$
\hat{a}_{d}[m]=-\frac{c f_{p r f}^{2}}{2 \pi f_{c}} \hat{c}_{r}[m], \quad m=1,2,3, . .
$$

where $\hat{c}_{r}$ is the estimated chirp rate at the $m$-th aperture.

\subsection{Description of the Targets}

In this chapter, we are estimating the vibration signatures and parameters of three types of vibrating targets. Each exhibits a different sinusoidal vibrational behavior. The first is a "top-hat" like chimney reflector, the second target is a rocking quad-corner reflector and the last one is sliding quad-corner reflector. The detailed description about the targets, types of motor, and accelerometer used to study the actual vibration can be found in [31].

\subsubsection{Top-hat chimney reflector}

This is one of the targets used for the experiment. It is a miniaturized structure with an exposed chimney, which conceals the vibrating source. The radar cross section of this target is modeled as a top-hat reflector. This design imitates a chimney that releases the gases to the atmosphere on the roof of the building. The motor is placed inside the cylindrical structure. A three-dimensional scheme of the complete structure is presented in Fig. (4.1).

The measured acceleration of the chimney driven by the actuating motor is presented in Fig. (4.2). The acceleration signal is noisy though we can distinguish the main frequency component of $5.56 \mathrm{~Hz}$. 


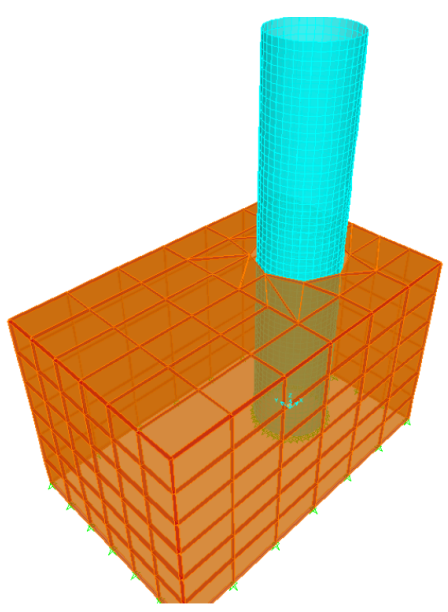

(a)
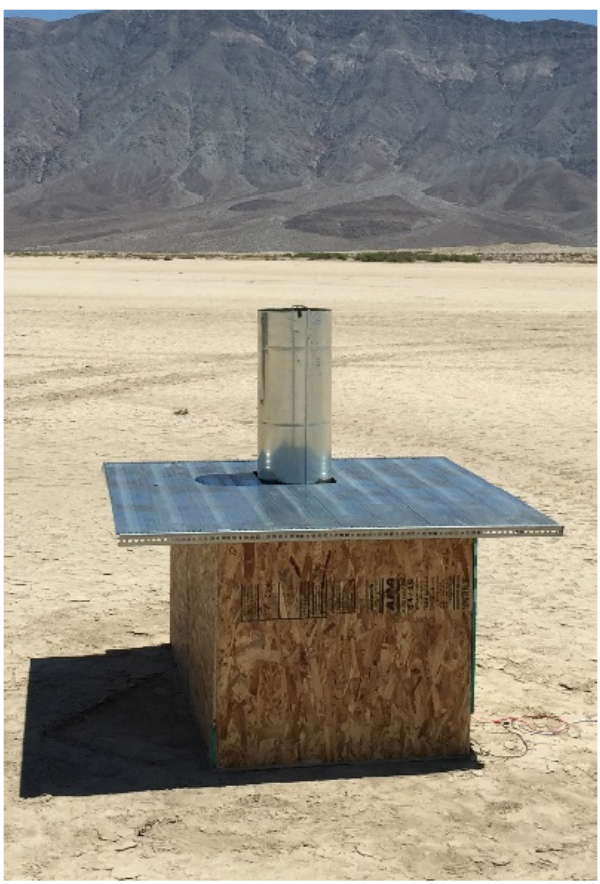

(b)

Figure 4.1: Graphical design of the top-hat chimney reflector. (a) 3-Dimensional perspective view , (b) Top-hat chimney reflector [31]

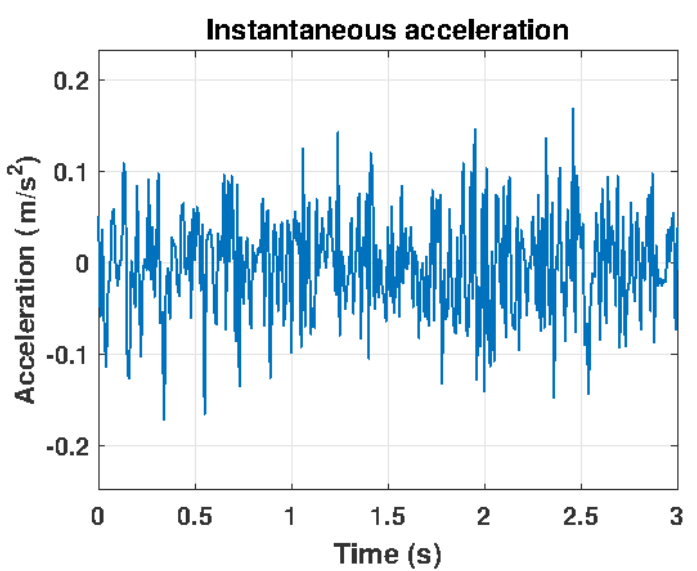

(a)

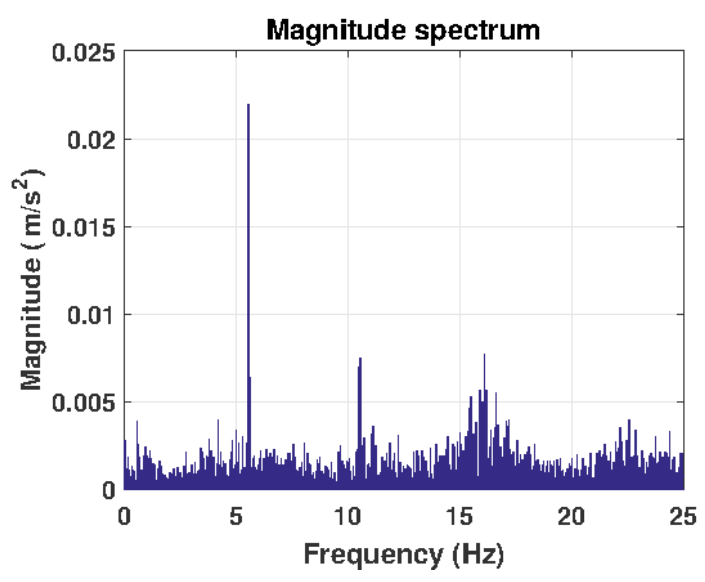

(b)

Figure 4.2: Vibrational signature of the chimney driven by the actuating motor. (a) Instantaneous acceleration waveform; (b) corresponding one-sided magnitude spectrum of (a).

\subsubsection{Rocking quad-corner reflector}

This is the second vibrating target used in the experiment as shown in Fig. (4.1)(a). The motor is placed on the base of the reflector, which exhibits a rocking movement when turned on. 
The acceleration measurement of this reflector is presented in Fig. (4.4). The vibration signature of rocking quad-corner reflector consists mainly of a sinusoidal waveform of $3.43 \mathrm{~Hz}$.

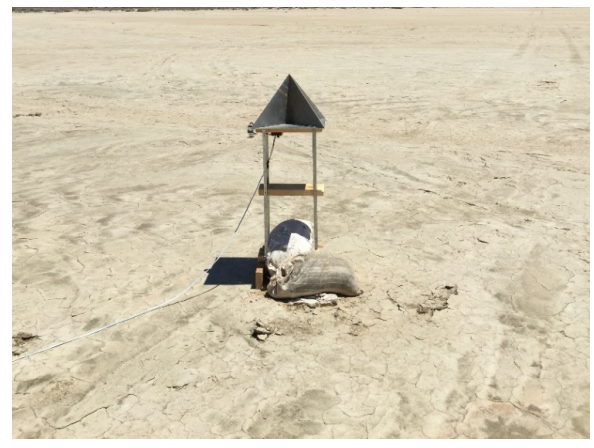

(a)

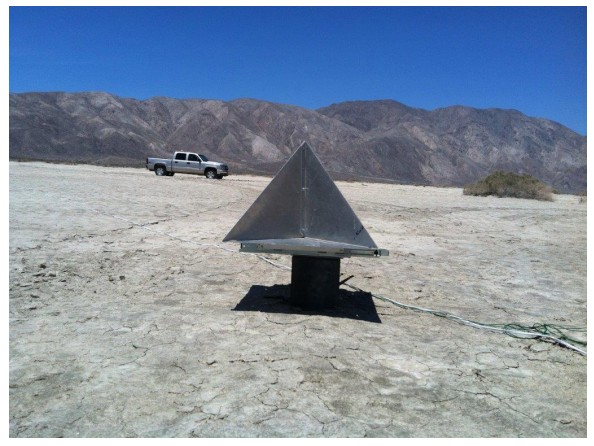

(b)

Figure 4.3: Targets without no concealed vibrating sources. (a) Rocking quad-corner reflector; (b) sliding quad-corner reflector [31].

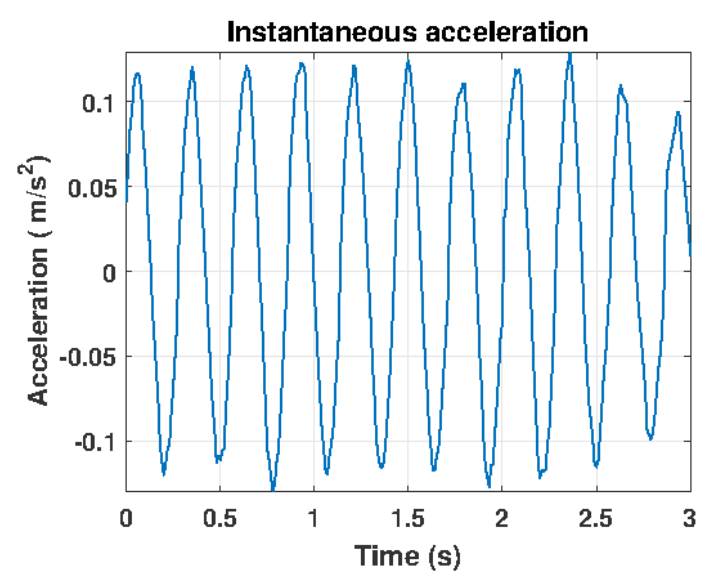

(a)

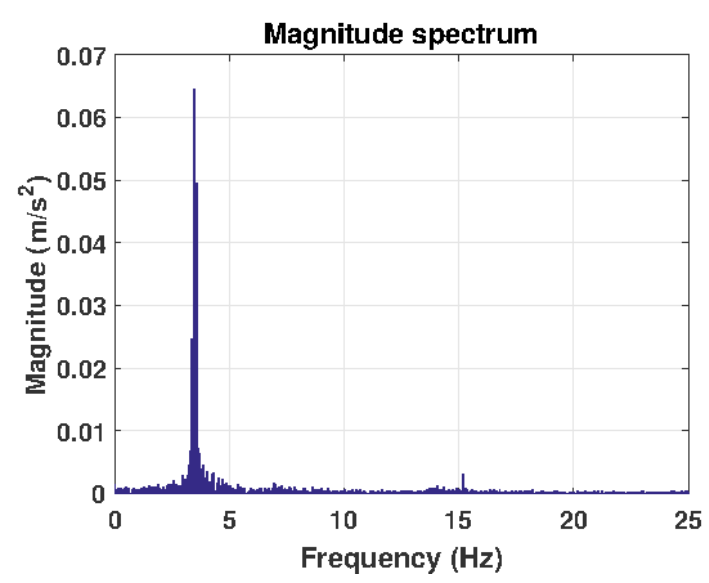

(b)

Figure 4.4: Vibrational signature of the rocking quad-corner reflector driven by the actuating motor. (a) Instantaneous acceleration waveform; (b) corresponding one-sided magnitude spectrum of (a).

\subsubsection{Sliding quad-corner reflector}

The third vibrating target in this experiment is a sliding quad-corner reflector that is depicted in Fig. (4.3)(b). The motor makes the quad-corner reflector slide over the rails.

The measured acceleration of this target is presented in Fig. (4.5). The vibration 
signature of this target consists of a sinusoidal waveform of $1.29 \mathrm{~Hz}$ and some harmonic components.

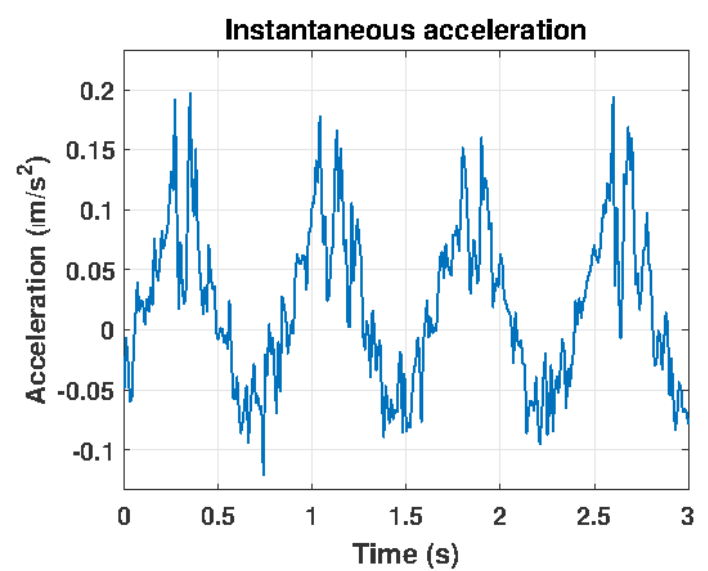

(a)

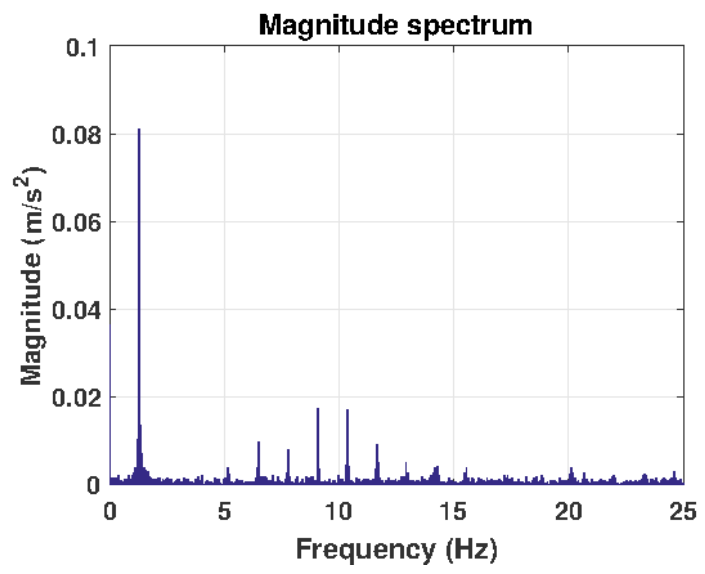

(b)

Figure 4.5: Vibrational signature of the sliding quad-corner reflector driven by the actuating motor. (a) Instantaneous acceleration waveform; (b) corresponding one-sided magnitude spectrum of (a).

\subsection{Estimation using the Analytical Expressions}

The SAR data was collected from a flight test in collaboration with General Atomics Aeronautical Systems Inc. (GA-ASI). The data were taken of the targets at 4-in resolution with the Lynx airborne $\mathrm{Ku}$-band SAR system [32]. The system parameters of the Lynx radar for 4-in resolution are detailed in Table 4.1. The separation between the targets was approximately 100". Fig. (4.6) shows the scheme with the relative position of the target. Fig. (4.7) shows the SAR image of the ground scene at 4-in resolution. As we can see in the same figure, the reflections of the

Table 4.1: SAR system parameters for the 4-in resolution data.

\begin{tabular}{l|l}
\hline \hline Parameter & Quantity \\
\hline Pixel dimension & $0.0858 \times 0.0885 \mathrm{~m}^{2}$ \\
\hline Nominal resolution & $0.1016 \times 0.1016 \mathrm{~m}^{2}$ \\
\hline Carrier frequency & $f_{c}=15 \mathrm{GHz}$ \\
\hline Length of the synthetic aperture & $L=176 \mathrm{~m}$ \\
\hline Plane velocity & $V_{a}=98 \mathrm{~m} / \mathrm{s}$ \\
\hline Effective pulse repetition frequency & $\hat{f}_{p r f}=185.43 \mathrm{~Hz}$ \\
\hline
\end{tabular}




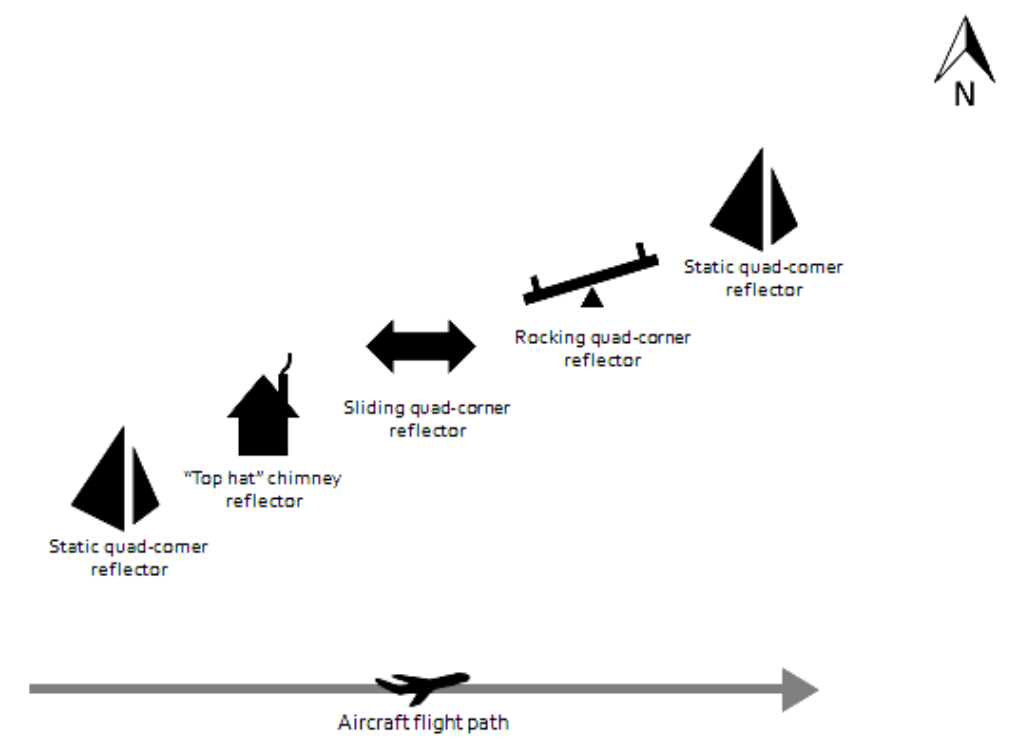

Figure 4.6: Layout of the targets on the ground scene [31].

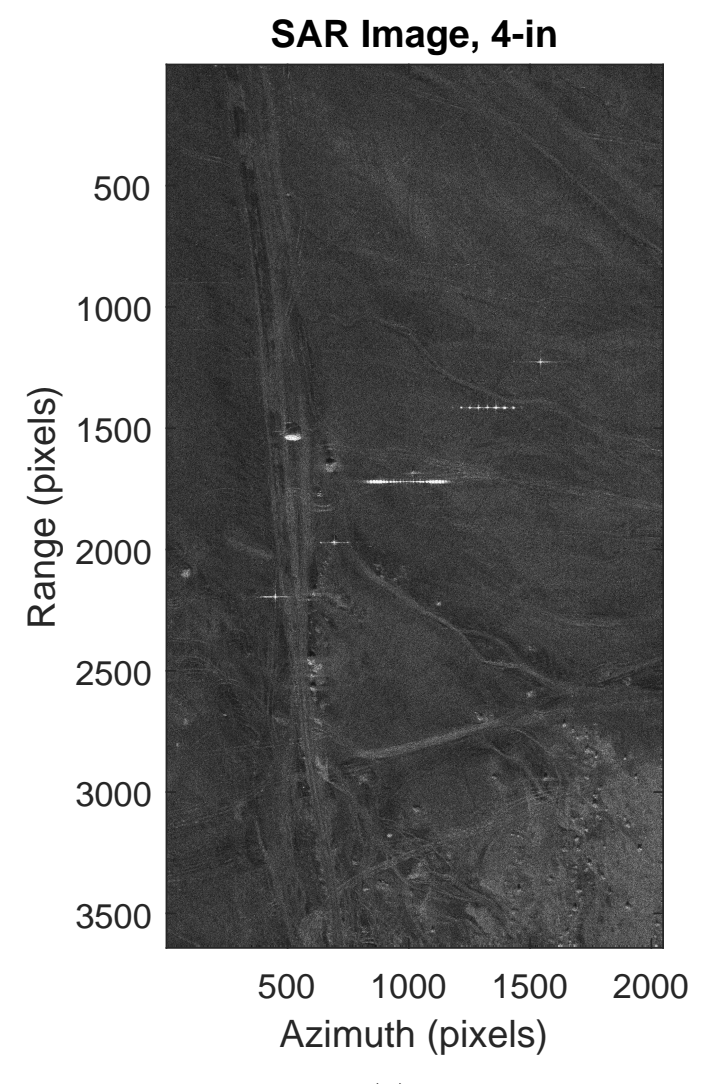

(a)

Figure 4.7: SAR image of the ground scene (a) 4-in resolution image

vibrating target presents a ghosting effect due to their movement. Usually, the size of this smearing effect is directly proportional to the amplitude of the target. 
This is the reason behind the strong reflection for the sliding quad-corner reflector, medium in the reflection for rocking the quad-corner reflector and the smallest in the top-hat chimney reflector. Even with the naked eye, the distinction between the targets can easily be seen by observing the smearing effects.

We now apply DFRFT techniques along with the analytical chirp rate estimator in order to distinguish the vibration signatures associated with the three vibrating target. The following parameters are used in the process:

Table 4.2: Parameters employed in the DFRFT-based vibrometry technique.

\begin{tabular}{l|c|c|c|c}
\hline \hline Target & Res. & Sub-aperture size (N) & M & Zoom-in \\
\hline Top-hat chimney & 4 -in & 32 & 256 & 2 \\
\hline Rocking quad-corner & 4 -in & 32 & 256 & 2 \\
\hline Sliding quad-corner & 4 -in & 80 & 256 & 2 \\
\hline
\end{tabular}

Here, the sub-aperture is the length of the signal in a moving-window. It is then zero padded to the length $\mathrm{M}$, typically a power of 2 , by adding an equal number of zeros on the either side of the signal. While applying the MA-CDFRFT algorithm, we also incorporate a zooming factor of 2 . We did not use the spectral peak interpolation technique with this data because as mentioned in previous chapter, it works best with the estimation of center frequency but not chirp rate parameter.

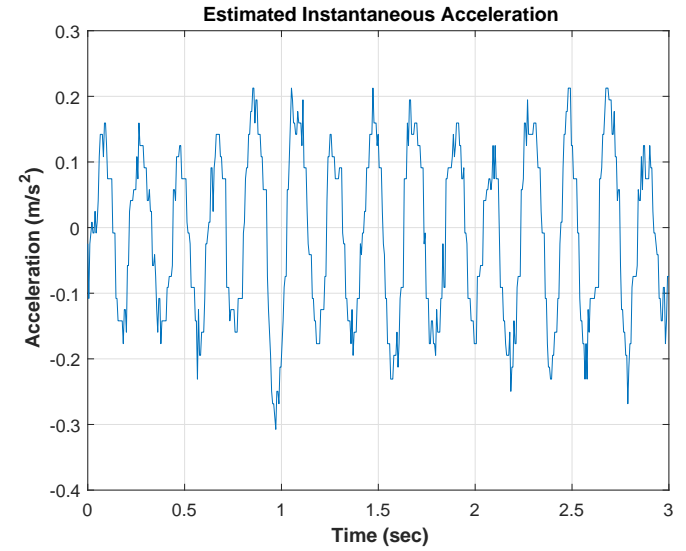

(a)

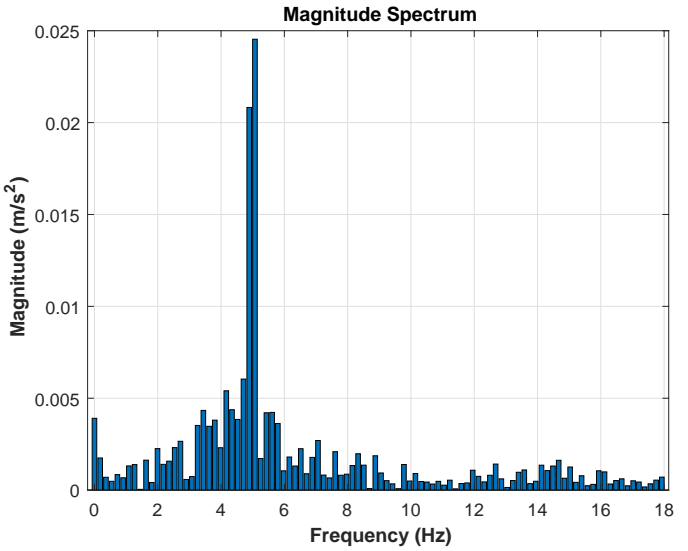

(b)

Figure 4.8: Estimated vibrational signature of the top-hat chimney reflector driven by the actuating motor. (a) Instantaneous acceleration waveform; (b) corresponding one-sided magnitude spectrum of (a). 
Fig. (4.8), (4.9) and (4.10) presents the results of applying the DFRFT-based vibrometry technique to data of 4 -in resolution. Table 4.3 details the parameters used in the algorithm for producing the results of Fig. (4.7) This technique allows us to identify the motion associated with the reflection from the targets. It also helps us differentiate between the targets by studying their instantaneous acceleration waveforms and respective magnitude spectrum. The actual acceleration waveform differs from the estimated one but we can see that their magnitude spectrum are similar.

Fig. (4.8) shows the estimated instantaneous acceleration and the magnitude spectrum of the top-hat chimney reflector. The acceleration waveform is similar to that obtained from the accelerometer. The frequency component of the highest magnitude corresponds to $5.07 \mathrm{~Hz}$ for this target compared to $5.56 \mathrm{~Hz}$ measured by accelerometer.

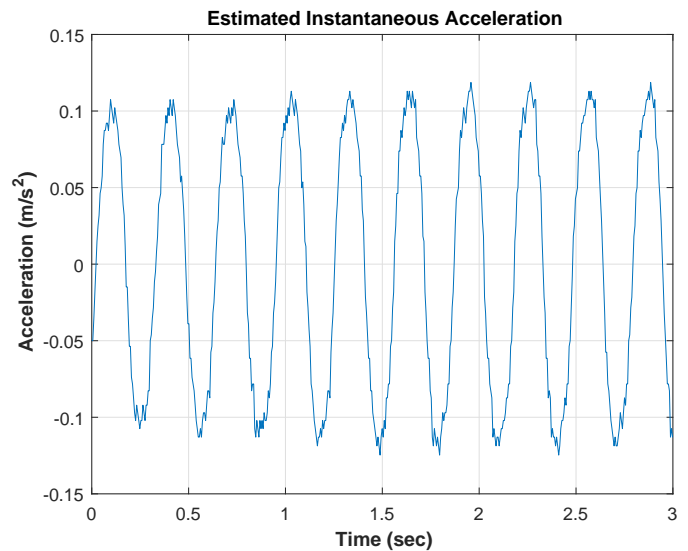

(a)

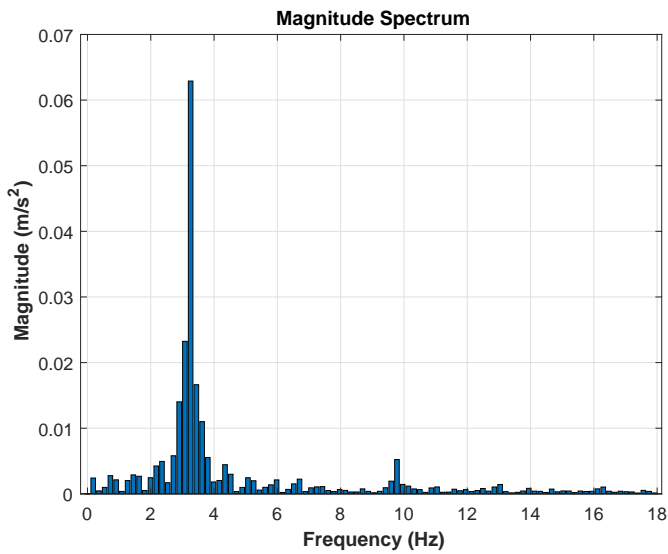

(b)

Figure 4.9: Estimated vibrational signature of the rocking quad-corner reflector driven by the actuating motor. (a) Instantaneous acceleration waveform; (b) corresponding one-sided magnitude spectrum of $(\mathrm{a})$.

Fig. (4.9) presents estimated acceleration waveform and its magnitude spectrum for the rocking quad-corner reflector target. The waveform is quite similar and so is the highest frequency component in the magnitude spectrum which is $3.26 \mathrm{~Hz}$ compared to $3.43 \mathrm{~Hz}$ read by accelerometer.

Fig. (4.10) shows the estimated acceleration and the magnitude spectrum of the sliding quad-corner target. The frequency component of the highest magnitude 


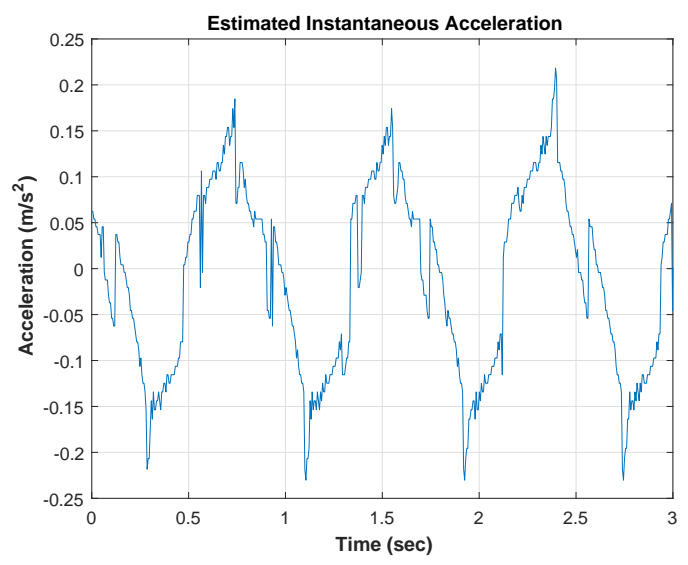

(a)

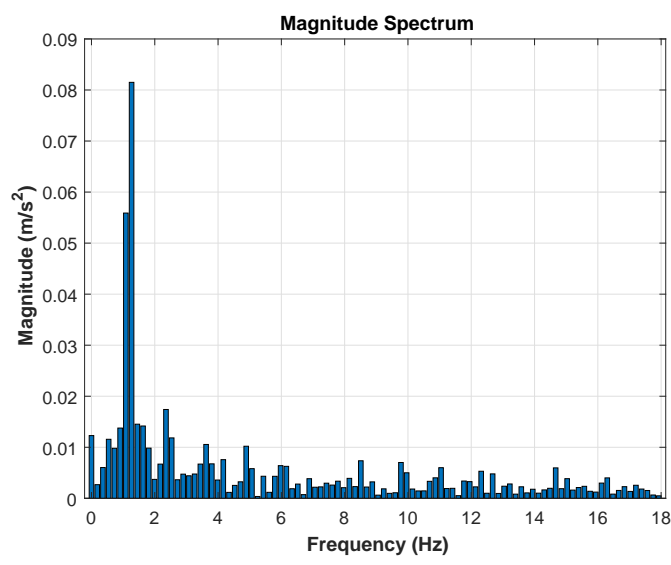

(b)

Figure 4.10: Estimated vibrational signature of the sliding quad-corner reflector driven by the actuating motor. (a) Instantaneous acceleration waveform; (b) corresponding one-sided magnitude spectrum of (a).

corresponds to $1.27 \mathrm{~Hz}$ compared to $1.29 \mathrm{~Hz}$ measured by accelerometer which acts as ground truth for the target.

In each case, it should be noted that the quality of the retrieved acceleration signals depends on how we accurately choose the parameters.

\subsection{Conclusion}

In this chapter, we studied the basics of SAR imagery and how they are formed. We also studied three different types of vibrating objects, each with their distinct vibration signature and acceleration waveforms. We used the analytical expression for the chirp parameters to calculate the chirp rate for the real SAR data and thus the underlying instantaneous acceleration from the peak-location obtained after applying the MA-DFRFT algorithm. The results obtained were very close to the ground truth that was measured from the accelerometer attached to the target itself. The limitations of this technique are mainly associated with the $f_{p r f}$ value of the SAR system and sub-aperture size that limits the maximum measurable frequency and the SNR and SCR that degrades the range-compressed phase history signal which is the staring point of this estimation technique. 
One major advantage of using analytical expressions is that the complexity of the approach for computing the estimates and the associated MSE is reduced, since an empirical mapping for each $N$ need not to be calculated. 


\section{Chapter 5}

\section{Conclusions and Future Work}

In this thesis, we studied the DFRFT based estimation of the chirp parameters of a real chirp signal in presence of noise. The main objective of this thesis was to quantify the accuracy of the chirp parameter estimates in terms of MSE over a range of SNR. First, we studied the distortion issues that arise in the transition from continuous FRFT to the DFRFT. We also looked at the sources of these errors and minimized the error by incorporating windowing effects into the QMFD approach. We further investigated extensions of this approach that resulted in a commuting matrix and associated eigenvectors with a reduced degree of distortion. We then looked at its effect on the parameter estimates in terms of MSE. We then shifted our focus to the peak-to-parameter mapping. We calculated the chirp parameter estimates using the peak location obtained from the magnitude spectrum of the MA-CDFRFT output. We further modified the existing DFRFT estimator to incorporate the "half-sample" shift. We then introduced various refinements to the estimators and finally proposed a combination that works best for center frequency and chirp rate separately. The next step was to apply the estimators to SAR vibrometry data. We studied the basics of SAR and the signal model associated with it. We looked at the three different types of vibrating targets briefly as well as the vibration signatures obtained from the accelerometer attached to it, which acts as ground truth measurements. We then compared the results from these estimators with the ground truth. 
The main findings of this thesis can be summarized as;

- Use of appropriate window to the eigenvalue sequence of the discrete operator results in improvement of the invertibility of the underlying peak-toparameter mapping and to the associated Mean Squared Errors of the chirp parameter estimates.

- Using the Kaiser windowed version of the truncated odd integer valued eigenvalue spectrum results in sharper peaks compared to the underlying DFRFT spectra in comparison to the boxcar windowed spectra which translated to a wider invertibility region for the underlying peak-to-parameter mapping. This improvement is more pronounced for smaller matrix sizes.

- The analytical expressions for the parameter estimates act as an improved version of the empirical estimator. Also combined with various refinements, these expressions gives results that are very close to the CRLB. For the center frequency estimate, the combination of zero padding and spectral peak interpolation is optimal while for the chirp rate, the combination of zero padding and CZT zoom is the optimal.

- These analytical expressions can be used with the SAR data. The instantaneous acceleration calculated from the chirp rate estimated using these expressions are very similar to the acceleration measured with accelerometer. Also, the highest frequency component of the magnitude spectrum of all three vibrating targets is very close to the ground truth.

\section{$5.1 \quad$ Future Work}

We have seen that the main bottleneck for this approach is the calculation of the peak itself. The MSE of the estimates gets very close to the CRLB when the size of CDFT is $M=2048$, however, the eigenvalue decomposition and thus generating the peak locations for this $M$ takes a long time. We think that the future extension 
of this thesis could be focused on calculating the efficient way for the eigenvalue decomposition which will result in faster MA-CDFRFT algorithm.

We also think that using the optimal window function with optimal parameters can help decrease the discretization errors even more, thus resulting in the much better estimates. So, extension to this thesis can be done in finding the best window function with optimal parameter for the lowest possible discretization error.

Since, the 2D MA-CDFRFT peaks are obtained from the DFT of $z_{k}[p]$ [20], for the chirp rate, it is a approximation of the MLE [33]. However, this is not the same case for center frequency. So, we think that, extension of this work can be done on developing a better approximation of the center frequency parameter.

There are also some other research dimensions that could be considered as extension of this thesis work other than application to the SAR data. Discrete version of the fractional sine and cosine transforms [34] has been proposed and significant progress in the are of image processing can be made. Some other applications can be in the areas of telecommunications and control systems. This work can be used in autonomous vehicles using radar to detect position and speed of the cars around it [35] [36]. 


\section{Appendix A}

\section{Parameter to Peak Mapping}

We look at the inverse of the peak-to-parameter mapping i.e. the mapping from the chirp parameter estimates to the ideal peak locations in the chirp rate vs center frequency plane. We use Eq. (3.10) to generate the inverse mapping. First, for the $r$-index,

$$
\begin{aligned}
& \hat{c}_{r}=-\frac{\pi}{N} \cot \left(\frac{2 \pi}{N}\left(r_{p}-\frac{1}{2}\right)\right) \\
\Rightarrow & -\frac{N}{\pi} \hat{c}_{r}=\cot \left(\frac{2 \pi}{N}\left(r_{p}-\frac{1}{2}\right)\right) \\
\Rightarrow & \tan \left(\frac{2 \pi}{N}\left(r_{p}-\frac{1}{2}\right)\right)=-\frac{\pi}{N \hat{c}_{r}} \\
\Rightarrow & \frac{2 \pi}{N}\left(r_{p}-\frac{1}{2}\right)=\tan ^{-1}\left(-\frac{\pi}{N \hat{c}_{r}}\right)=-\tan ^{-1}\left(\frac{\pi}{N \hat{c}_{r}}\right) \\
\Rightarrow & r_{p}-\frac{1}{2}=-\frac{N}{2 \pi} \tan ^{-1}\left(\frac{\pi}{N \hat{c}_{r}}\right) \\
\Rightarrow & r_{p}=\frac{1}{2}-\frac{N}{2 \pi} \tan ^{-1}\left(\frac{\pi}{N \hat{c}_{r}}\right)
\end{aligned}
$$


For the $k$-index,

$$
\begin{aligned}
\hat{\omega}_{c} & =\frac{2 \pi}{N}\left(k_{p}-\frac{N}{2}\right) \csc \left(\frac{2 \pi}{N}\left(r_{p}-\frac{1}{2}\right)\right) \\
\Rightarrow \hat{\omega}_{c} & =\frac{2 \pi}{N}\left(k_{p}-\frac{N}{2}\right) \csc \left(\frac{2 \pi}{N}\left(\frac{1}{2}-\frac{N}{2 \pi} \tan ^{-1}\left(\frac{\pi}{N \hat{c}_{r}}\right)-\frac{1}{2}\right)\right) \\
\Rightarrow & \hat{\omega}_{c}=\frac{2 \pi}{N}\left(k_{p}-\frac{N}{2}\right) \csc \left(-\tan ^{-1}\left(\frac{\pi}{N \hat{c}_{r}}\right)\right) \\
\Rightarrow \hat{\omega}_{c}= & -\csc \left(\tan ^{-1}\left(\frac{\pi}{N \hat{c}_{r}}\right)\right) \frac{2 \pi}{N}\left(k_{p}-\frac{N}{2}\right) \\
\Rightarrow & \hat{\omega}_{c}=\sin \left(\tan ^{-1}\left(\frac{\pi}{N \hat{c}_{r}}\right)\right)=\frac{2 \pi}{N}\left(k_{p}-\frac{N}{2}\right) \\
\Rightarrow & \frac{N \hat{\omega}_{c}}{2 \pi} \sin \left(\tan ^{-1}\left(\frac{\pi}{N \hat{c}_{r}}\right)\right)=\left(k_{p}-\frac{N}{2}\right) \\
\Rightarrow & k_{p}=\frac{N}{2}+\frac{N \hat{\omega}_{c}}{2 \pi} \sin \left(\tan ^{-1}\left(\frac{\pi}{N \hat{c}_{r}}\right)\right) \\
&
\end{aligned}
$$

From Eq. (A.1) and (A.2), we have the ideal location of the peak. We can see that, the $r$-index only depends on the chirp rate, however, the $k$-index depends on center frequency as well as chirp rate. In the inverse mapping, the parameter estimates are dependent on the $\tan ^{-1}\left(\frac{\pi}{N \hat{c}_{r}}\right)$ factor but the arctangent is not invertible (not one-to-one) for all ranges which means that there is going to be a set of values of $\left(\hat{\omega}_{c}, \hat{c}_{r}\right)$ for which the peak location is the same and vice versa. 


\section{Appendix B}

\section{Parameter Estimation using}

\section{Analytical expressions and}

\section{Subspace Decomposition}

Subspace methods for chirp parameter estimation were developed in [37] [5], where the two dimensional MA-CDFRFT representation is projected into each dimension using the $p$-norm using the cross-hair approach and inverse discrete Fourier transformed slices to yield time-domain quantities. Traditional subspace methods such as MUSIC, eigenvector, or minimum norm approaches can then be applied to the transformed projections. Prior work in [5] [38] used the empirical peak to parameter mappings to estimate the chirp parameter. Here we use the analytical expressions for the peak to parameter mapping to evaluate the performance of the subspace approach. We specifically look at the minimum-norm approach which produced the best results in the empirical mapping application.

To find the peak location from the subspace method, first, the coordinates of the maximum index along both $r$-axis and $k$-axis is calculated. Then, two timeseries, one for chirp rate and another for center frequency are obtained using the inverse FFT at the maximum location calculated above. These time series are then projected to $R=2048$ subspace points using a 3-norm projection and the minimum norm approach to obtain the pseudo-spectrum. The maximum value 


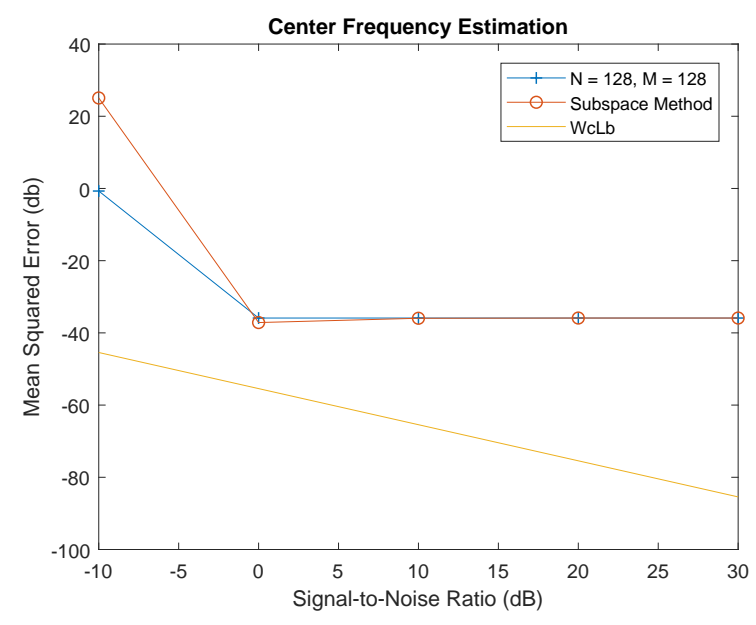

(a)

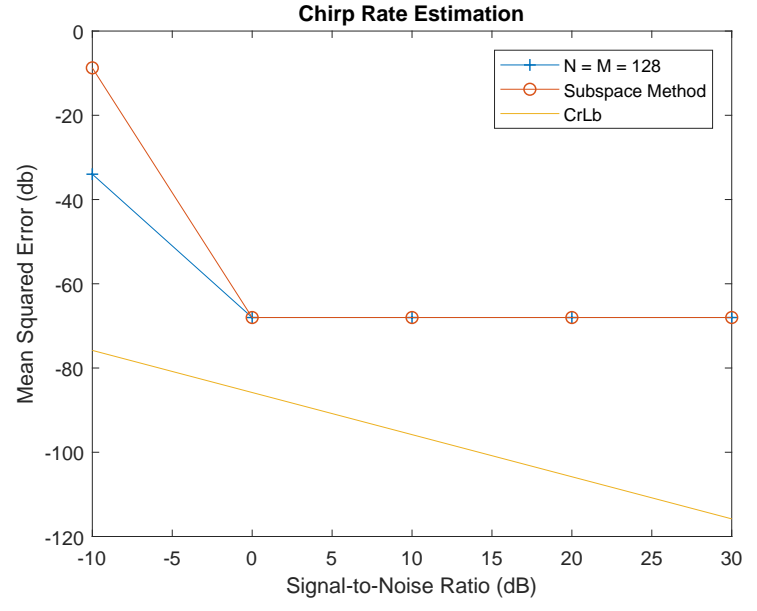

(b)

Figure B.1: Subspace methods and analytical expressions: (a,b) comparison of the MSE associated with the analytical formulas with the min-norm subspace approach with $N=M=128$ using a 3 -norm projection, $L=128$-order correlation matrices and $R=2048$-pt FFT within the subspace method.

in the pseudo-spectrum is sorted to find different peaks. These locations are then scaled back to the original signal length to get the actual peak location. Now, this peak location is fed into the analytical expressions in Eq. (3.10) to calculate the chirp parameter estimates. Fig. (B.1) depicts the application of the analytical formulas in conjunction with the minimum norm approach. Since, subspace method gives fine resolution of the peak only, we can see that there is not much improvement in the MSE of the chirp parameters, instead it appears to be worse for lower SNR values. This is attributable to the fact that the cross-hair approach used in the subspace method assumes separable variables, whereas the estimator in the analytical expressions are inherently coupled. 


\section{Bibliography}

[1] C. Johnson, W. Sethares, and A. Klein, Software Receiver Design: Build Your Own Digital Communications System in Five Easy Steps. 2011.

[2] W. Knight, R. Pridham, and S. Kay, "Digital signal processing for sonar", Proceedings of the IEEE, vol. 69, no. 11, pp. 1451-1506, 1981.

[3] B. Widrow and S. Stearns, "Adaptive signal processing", Englewood Cliffs, NJ, Prentice-Hall, Inc., 1985, 491 p., vol. 1, 1985.

[4] Q. Wang, M. Pepin, R. J. Beach, R. Dunkel, T. Atwood, B. Santhanam, W. Gerstle, A. W. Doerry, and M. M. Hayat, "SAR-based Vibration Estimation using the Discrete Fractional Fourier Transform", IEEE Trans. Geoscience and Remote Sensing, Vol. 50, No. 10, pp. 4145-4156, 2012.

[5] D. J. Peacock and B. Santhanam, "Multicomponent Subspace Chirp Parameter Estimation Using Discrete Fractional Fourier Analysis", Proc. of IASTED Conference on SIP-2011, pp. 326-333, Dec., 2011.

[6] V. Namias, "The fractional Order Fourier Transform and its application to Quantum mechanics", J. Inst. Mathematics Application, 25, pg. 241-265, 1980 .

[7] B. Santhanam and J.H. McClellan, The Discrete Rotational Fourier Transform, IEEE Transactions In Signal Processing, Vol. 44, No. 4, April 1996.

[8] J.H. McClellan and T.W. Parks, "Eigenvalues And Eigenvectors Of The Discrete Fourier Transformation", IEEE Transactions on Audio and Electromagnetics, Vol. 20, No. 1, March 1972.

[9] G. Strang, Linear Algebra And Its Application, Academic Press, New York, 1995.

[10] R.M. Liang and K.S. Arun, "Parameter estimation for superimposed chirp signals", Proc. of ICASSP, 1992, 273-276.

[11] B. Friedlander, J.M. Francos, "Estimation of Amplitude and Phase Parameters of Multicomponent Signals", IEEE Transactions on Signal Processing, Vol. 43, No. 4, April 1995.

[12] A. V. Oppenhein, R. W. Schafar, "Discrete Time Signal Processing", Prentice Hall Press Upper Saddle River, 2009. 
[13] Balu Santhanam and T. S. Santhanam, "On Discrete Gauss-Hermite Functions and Eigenvectors of the Discrete Fourier Transform", Signal Processing, Vol. 88, No. 6, pp. 2738 - 2746,November 2008.

[14] B. Dickinson and K. Steiglitz, "Eigenvectors and Functions of the Discrete Fourier Transform", IEEE Trans. Sig. Process., Vol. 30, No. 1, pp. 25 - 31, February 1982.

[15] F. Grunbaum, "The eigenvectors of the Discrete Fourier transform: A version of the Hermite functions", Journal of Mathematical Analysis and Applications, Vol. 88, No. 2, pp. 355-363, August 1982.

[16] F. J. Harris, "On the Use of Windows for Harmonic Analysis with the Discrete Fourier Transform", Proc. of IEEE, Vol. 66, pp. 51-83, 1978.

[17] B. Santhanam, T. Santhanam, S. Mandal, "On the Effects of Windowing on the Discretization of the Fractional Fourier Transform", Asilomar Conference, Nov 2017.

[18] T. Santhanam and B. Santhanam, "The Discrete Fourier Transform and the Quantum Mechanical Oscillator in a Finite-Dimensional Hilbert Space", Journal Of Physics A: Theoretical, Vol. 42, pp. 205303, May 2009.

[19] Ishwor Bhatta and Balu Santhanam, "A Comparative Study Of Commuting Matrix Approaches For The Discrete Fractional Fourier Transform", Proc. of IEEE Signal Processing and SP Education Workshop, pp. 103-108, 2015.

[20] J.G. Vargas-Rubio and B. Santhanam, On The Multi angle Centered Discrete Fractional Fourier Transform, IEEE Signal Processing Letters, Vol. 12, No. 4, April 2005.

[21] M.A. Richards, "Fundamentals of Radar Signal Processing", New York: McGraw, 2011.

[22] C. Candan, "A Method for Fine Resolution Frequency Estimation From Three DFT Samples", IEEE Signal Processing Letters, Vol. 18, No. 6, June 2011.

[23] B.G. Quinn, "Estimation of Frequency by interpolation using Fourier coefficients", IEEE Trans. Signal Processing, Vol. 42, No. 5, pp. 1264-1268, May 1994.

[24] R. Gens, J. L. V. Genderen," "Review article SAR interferometry-issues, techniques, applications", International Journal of Remote Sensing 17(10), 18031835, 1996.

[25] A. H. S. Solberg, G. Storvik, R. Solberg, and E. Volden, "Automatic detection of oil spills in ERS SAR images", IEEE Transactions on Geoscience and Remote Sensing 37, 1916-1924, Jul 1999.

[26] G. W. Stimson "Introduction to Airborne RADAR", Chapter 1 pp. 13. 
[27] R. K. Raney, "Synthetic aperture imaging radar and moving targets," IEEE Transactions on Aerospace and Electronic Systems AES-7, 499-505, May 1971.

[28] Q. Wang, m. Pepin, A. Wright,R. Dunkel, T. Atwood, B. Santhanam, W. Gerstle, A. W. Doerry, and M. M. Hayat, "Reduction of vibration-induced artifacts in synthetic aperture radar imagery," IEEE Transactions on Geoscience and Remote Sensing 52, 3063-3073, June 2014.

[29] J. Adebello, B. Santhanam, "Clutter suppression in synthetic aperture radar targets using the DFRFT and subspace methods with rank reduction", in 2015 49th Asilomar Conference on Signals, Systems and Computers, 16691673, Nov 2015.

[30] Q. Wang, B. Santhanam, M. Pepin, M. M. Hayat, "Performance analysis on synthetic aperture radar- based vibration estimation in clutter", Signals, Systems and Computers (ASILOMAR), 2012 Conference Record of the Forty Sixth Asilomar Conference on, 217-221, Nov 2012.

[31] F. Pérez, J. B. Campbell, M. Jaramillo, R. Dunkel, T. Atwood, A. Doerry, W. H. Gerstle, B. Santhanam, M. M. Hayat, "Exploiting synthetic aperture radar imagery for retrieving vibration signatures of concealed machinery", Proc. SPIE 9829, Radar Sensor Technology XX, 982903, 12 May 2016.

[32] S. I. Tsunoda, F. Pace, J. Stence, M. Woodring, W. H. Hensley, A. Doerry, B. C. Walker, "Lynx: a high-resolution synthetic aperture radar", 1999.

[33] Y. Liao, "Phase and Frequency Estimation: High Accuracy and LowComplexity Techniques", May 2011.

[34] S.C. Pei, M.H. Yeh, "The Discrete Fractional Cosine And Sine Transforms", IEEE Transactions of Signal Processing, Vol. 49, No.6, Pages 1198 - 1207, (2001)

[35] S. Park and Y. Kim and E. T. Matson and A. H. Smith, "Accessible synthetic aperture radar system for autonomous vehicle sensing", IEEE Sensors Applications Symposium (SAS), 2016.

[36] D. Clarke and D. Andre and F. Zhang, "Synthetic aperture radar for lane boundary detection in driver assistance systems", IEEE International Conference on Multisensor Fusion and Integration for Intelligent Systems (MFI), 2016.

[37] B. Santhanam and M. Hayat, "On A Pseudo-Subspace Framework For Discrete Fractional Fourier Transform Based Chirp Parameter Estimation", Processing of IEEE DSP/SPE Workshops, Pages 360 - 363, 2011.

[38] D. J. Peacock, B. Santhanam, "Comparison of Discrete Fractional Fourier Transform Bases for Wideband Chirp Parameter Estimation", Proc. of IEEE DSP/SP Education Workshop, pp. 103-108, Aug 2015. 